\title{
Be/X-ray Binaries
}

\author{
Pablo Reig ${ }^{1,2}$
}

\begin{abstract}
The interest in $\mathrm{X} / \gamma$-ray Astronomy has grown enormously in the last decades thanks to the ability to send X-ray space missions above the Earth's atmosphere. There are more than half a million X-ray sources detected and over a hundred missions (past and currently operational) devoted to the study of cosmic $\mathrm{X} / \gamma$ rays. With the improved sensibilities of the currently active missions new detections occur almost on a daily basis. Among these, neutron-star X-ray binaries form an important group because they are among the brightest extra-solar objects in the sky and are characterized by dramatic variability in brightness on timescales ranging from milliseconds to months and years. Their main source of power is the gravitational energy released by matter accreted from a companion star and falling onto the neutron star in a relatively close binary system.

Neutron-star X-ray binaries divide into high-mass and low-mass systems according to whether the mass of the donor star is above $\sim 8$ or below $\sim 2 \mathrm{M}_{\odot}$, respectively. Massive X-ray binaries divide further into supergiant $\mathrm{X}$-ray binaries and $\mathrm{Be} / \mathrm{X}$-ray binaries depending on the evolutionary status of the optical companion. Virtually all Be/X-ray binaries show X-ray pulsations. Therefore, these systems can be used as unique natural laboratories to investigate the properties of matter under extreme conditions of gravity and magnetic field.

The purpose of this work is to review the observational properties of Be/X-ray binaries. The open questions in Be/X-ray binaries include those related to the Be star companion, that is, the so-called "Be phenomenon", such as, timescales associated to the for-

Pablo Reig

${ }^{1}$ IESL, Foundation for Reseach and Technology-Hellas, 71110, Heraklion, Greece.

${ }^{2}$ Physics Department, University of Crete, 71003, Heraklion, Greece.
\end{abstract}

mation and dissipation of the equatorial disc, massejection mechanisms, $\mathrm{V} / \mathrm{R}$ variability, and rotation rates; those related to the neutron star, such as, mass determination, accretion physics, and spin period evolution; but also, those that result from the interaction of the two constituents, such as, disc truncation and mass transfer. Until recently, it was thought that the Be stars' disc was not significantly affected by the neutron star. In this review, I present the observational evidence accumulated in recent years on the interaction between the circumstellar disc and the compact companion. The most obvious effect is the tidal truncation of the disc. As a result, the equatorial discs in $\mathrm{Be} / \mathrm{X}$ ray binaries are smaller and denser than those around isolated Be stars.

Keywords X-rays: binaries - stars: neutron - stars: binaries close -stars: emission line, Be

\section{Definition and classification of X-ray binaries}

In very general terms, one can simply define X-ray binaries as systems that consist of a compact object orbiting an optical companion. They are "close" binary systems because there exists a transfer of mass from the optical component to the compact object. By "optical companion" it is understood that nuclear burning is still taking place in its interior. Figure 1 shows a tree-diagram depicting all the different subsystems that comprise the generic group of X-ray binaries.

In referring to the two components in an X-ray binary one should be careful and learn which is the subject of investigation as the same name can be used to mean different components. In massive X-ray binaries, the most massive star is normally termed primary whereas the less massive one is called secondary. In lowmass systems, the term primary refers to the neutron 


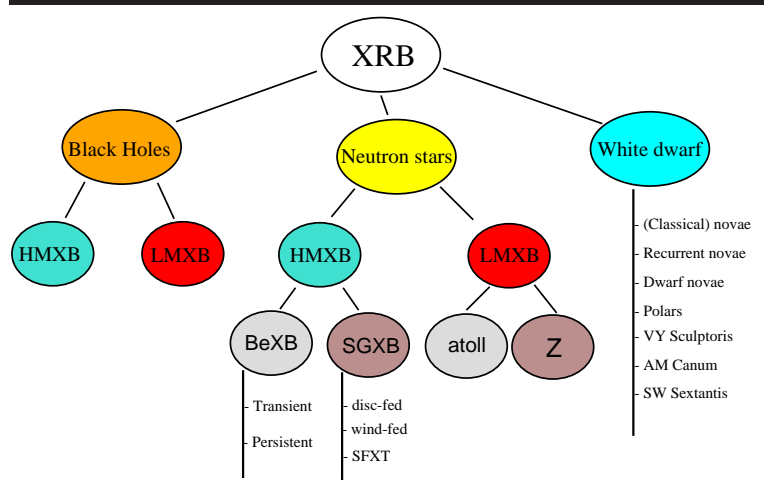

Fig. 1 Classification of X-ray binaries.

star while the word secondary is reserved for the latetype companion. Other names also used are "optical" or "donor" for the larger star and "compact", "gainer" or "accreting" for the denser companion.

They admit several classification schemes depending upon whether the emphasis is put on the type of the compact companion or the physical properties of the optical star. X-ray binaries divide up into blackhole systems, neutron star X-ray binaries or cataclysmic variables (if the compact object is a white dwarf). Nevertheless, the term "X-ray binaries" is normally reserved to designate binaries with neutron stars.

\subsection{High-mass X-ray binaries}

Neutron-star X-ray binaries are divided up into highmass (HMXBs) and low-mass (LMXBs) X-ray binaries depending on the spectral type of the mass donor, as this feature determines the mode of transferring mass to the compact object and the environment surrounding the X-ray source. HMXBs contain early-type (O or B) companions, while the spectral type of the optical star in LMXBs is later than A. HMXBs are strong emitters of X-ray radiation. Sometimes they appear as the brightest objects of the X-ray sky. The high-energy radiation is produced as the result of accretion of matter from the optical companion onto the neutron star. The term accretion refers to the gradual accumulation or deposition of matter onto the surface of an object under the influence of gravity. If the accreting object is a neutron star (or black hole), then matter falls down onto an enormous well of gravitational potential and is accelerated to extremely high velocities. When the matter reaches the surface of the neutron star, it is rapidly decelerated and the free-fall kinetic energy radiated away as heat which is available to power the X-ray source.

The luminosity class serves to subdivide HMXBs into $\mathrm{Be} / \mathrm{X}$-ray binaries (BeXB), when the optical star is a dwarf, subgiant or giant OBe star (luminosity class III,
IV or V) and supergiant X-ray binaries (SGXBs), if they contain a luminosity class I-II star.

In SGXBs, the optical star emits a substantial stellar wind, removing between $10^{-6}-10^{-8} \mathrm{M}_{\odot} \mathrm{yr}^{-1}$ with a terminal velocity up to $2000 \mathrm{~km} \mathrm{~s}^{-1}$. A neutron star in a relatively close orbit will capture some fraction of this wind, sufficient to power a bright X-ray source. If mass transfer occurs via Roche lobe overflow, then the X-ray emission is highly enhanced and an accretion disc is formed around the neutron star. At present, there is known only one disc-fed SGXB in the Galaxy (Cen X-3) and three in total (SMC X-1 and LMC X-4), while there are about a few tens of wind-fed SGXBs. Because of their brightness and persistent X-ray emission, SGXBs were the first to be discovered. They were initially thought to represent the dominating population of HMXBs, whereas BeXB were considered atypical cases. Hence, the name classical or standard was given to SGXBs.

In BeXB, the optical companion is a Be star. Be stars are non-supergiant fast-rotating B-type and luminosity class III-V stars which at some point of their lives have shown spectral lines in emission, hence the qualifier " $e$ " in their spectral types (Porter \& Rivinius 2003; Balona 2000; Slettebak 1988). The best studied lines are those of hydrogen (Balmer and Paschen series) but Be stars may also show He, Fe in emission (see e.g Hanuschik 1996). They also show an amount of IR radiation than is larger than that expected from an absorption-line B star of the same spectral type. This extra long-wavelength emission is known as infrared excess.

The origin of the emission lines and infrared excess in $\mathrm{BeXB}$ is attributed to an equatorial disc, fed from material expelled from the rapidly rotating Be star in a manner that it is not yet understood (Porter \& Rivinius 2003). During periastron, the neutron star passes close to this disc, sometimes may even go through it causing major disruption. A large flow of matter is then accreted onto the compact object. The conversion of the kinetic energy of the in-falling matter into radiation powers the $\mathrm{X}$ rays. BeXB have large orbital periods and by definition they are non-supergiant systems. Hence, the Be star is well within the Roche lobe. However, transient Roche lobe overflow may occur during periastron passage in systems with large eccentric orbits or during giant X-ray outbursts when a large fraction of the Be star's disc is believed to be accreted.

Most BeXB are transient systems and present moderately eccentric orbits $(e \gtrsim 0.3)$, although persistent sources also exist (Reig \& Roche 1999). Persistent $\mathrm{BeXB}$ differ from transient BeXB in that they display much less X-ray variability (no large outbursts are detected), lower luminosity $\left(L_{x} \lesssim 10^{35}\right.$ erg s$\left.{ }^{-1}\right)$, contain 


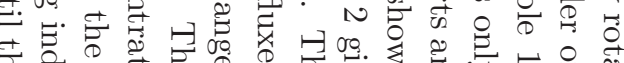
ज. क ज.

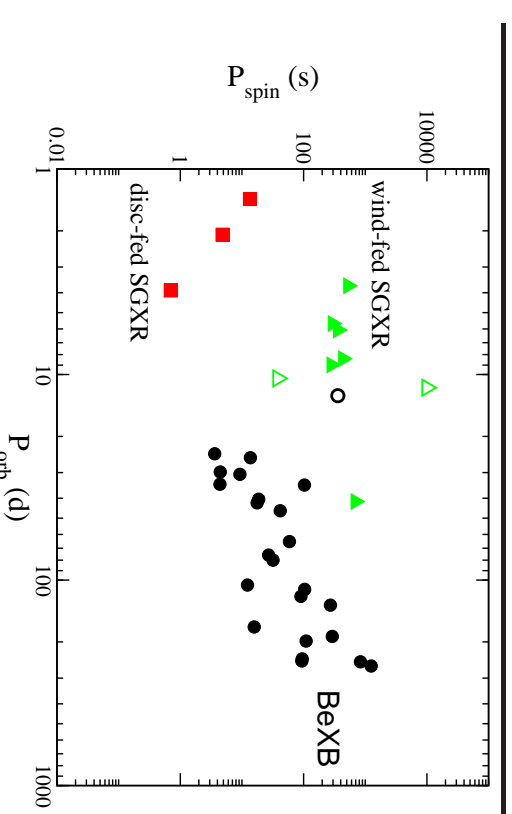

Table 1 List of galactic BeXB. Only systems with known optical counterparts and well-established optical/X-ray behaviour are included

\begin{tabular}{|c|c|c|c|c|c|c|c|c|c|c|c|}
\hline $\mathrm{X}$-ray name & Optical Ctp. & RA (J2000) & Dec $(J 2000)$ & Spec. type & $\mathrm{V}$ & $\mathrm{J}$ & $E(B-V)$ & $P_{\text {spin }}(\mathrm{s})$ & $P_{\text {orb }}(\mathrm{d})$ & $\mathrm{e}$ & $(\mathrm{kpc})$ \\
\hline $4 \mathrm{U} 0115+634$ & V635 Cas & 011831.90 & +634424.0 & B0.2 Ve & $14.8-15.5$ & $10.8-12.3$ & 1.55 & 3.6 & 24.3 & 0.34 & 8 \\
\hline IGR J01363+6610 & - & 013618.00 & +66 1036.0 & B1 IV-Ve & 13.3 & 10 & 1.61 & - & - & - & 2 \\
\hline RX J0146.9+6121 & LS I +61 235 & 014700.17 & +612123.7 & $\mathrm{~B} 1 \mathrm{Ve}$ & 11.2 & 10 & 0.93 & 1412 & 330 & - & 2.3 \\
\hline IGR J01583+6713 & - & $\begin{array}{llll}01 & 58 & 18.20\end{array}$ & +671326.0 & B2IVe & 14.4 & 11.5 & 1.46 & 19692 & - & - & 4 \\
\hline RX J0240.4+6112 & LS I +61303 & 024031.67 & +611345.6 & B0.5 Ve & 10.7 & 8.8 & 0.75 & - & 26.45 & 0.54 & 3.1 \\
\hline V $0331+530$ & BQ Cam & 033459.89 & +531023.6 & O $8-9 \mathrm{Ve}$ & $15.1-15.8$ & $11.4-12.2$ & 1.9 & 4.4 & 34.3 & 0.3 & 7 \\
\hline 4U 0352+309 (X Per) & HD 24534 & 035523.08 & +310245.0 & O9.5IIIe-B0Ve & $6.1-6.8$ & $5.7-6.5$ & 0.4 & 837 & 250 & 0.11 & 1.3 \\
\hline RX J0440.9+4431 & $\mathrm{LS} \mathrm{V}+4417$ & 044059.32 & +443149.3 & $\mathrm{~B} 0.2 \mathrm{Ve}$ & 10.8 & 9.2 & 0.65 & 203 & - & - & 3.3 \\
\hline 1A $0535+262$ & V725 Tau & 053854.57 & +261856.8 & B0 IIIe & $8.9-9.6$ & $7.7-8.5$ & 0.75 & 105 & 111 & 0.47 & 2.4 \\
\hline IGR J06074+2205 & - & $06 \quad 0724.00$ & +220500.0 & $\mathrm{~B} 0.5 \mathrm{~V}$ & 12.3 & 10.5 & 1.88 & - & - & - & 5 \\
\hline XTE J0658-073 & - & $0658 \quad 42.00$ & -071100.0 & O9.7 Ve & 12.1 & 9.7 & 1.19 & 160.4 & - & - & 3.9 \\
\hline $4 \mathrm{U} 0726-260$ & V441 Pup & 072853.60 & $\begin{array}{lll}-26 & 06 & 29.0\end{array}$ & O8-9Ve & 11.6 & 10.4 & 0.73 & 103.2 & 34.5 & - & 6 \\
\hline RX J0812.4-3114 & LS 992 & $\begin{array}{lll}08 & 12 & 28.84\end{array}$ & $\begin{array}{lll}-31 & 14 & 52.2\end{array}$ & B0.2 III-IVe & 12.4 & $11.2-12.0$ & 0.65 & 31.89 & 80 & - & 9 \\
\hline GS $0834-430$ & - & 083555.00 & $\begin{array}{llll}-43 & 11 & 06.0\end{array}$ & B0-2 III-Ve & 20.4 & 13.3 & 4.0 & 12.3 & 105.8 & 0.12 & 5 \\
\hline GRO J1008-57 & - & 100946.00 & $\begin{array}{lll}-58 & 17 & 30.0\end{array}$ & O9e-B1e & 15.3 & 10.9 & 1.9 & 93.5 & 247.5 & 0.66 & 2 \\
\hline RX J1037.5-5647 & LS 1698 & 103735.50 & $\begin{array}{llll}-56 & 48 & 11.0\end{array}$ & B0 III-Ve & 11.3 & - & 0.75 & 862 & - & - & 5 \\
\hline 1A $1118-615$ & Hen 3-640 & 112057.21 & $\begin{array}{lll}-61 & 55 & 00.3\end{array}$ & O9.5 III-Ve & 12.1 & 9.6 & 0.92 & 405 & - & - & 5 \\
\hline 4U $1145-619$ & V801 Cen & 114800.02 & $\begin{array}{lll}-62 & 12 & 24.9\end{array}$ & B1 Vne & 9.3 & 8.7 & 0.29 & 292.4 & 187.5 & $>0.5$ & 3.1 \\
\hline $4 \mathrm{U} 1258-613$ & GX 304-1 & $1301 \quad 17.20$ & $\begin{array}{llll}-61 & 36 & 07.0\end{array}$ & B2 Vne & 13.5 & 9.8 & 2.0 & 272 & 132.5 & $>0.5$ & 2.4 \\
\hline $2 \mathrm{~S} 1417-624$ & - & 142112.80 & -624154.0 & $\mathrm{~B} 1 \mathrm{Ve}$ & 17.2 & 13.3 & 2.0 & 17.6 & 42.12 & 0.45 & 10 \\
\hline GS $1843+00$ & - & $1845 \quad 36.90$ & +005148.2 & B0-2 IV-Ve & 20.9 & 13.7 & 2.5 & 29.5 & - & - & $>10$ \\
\hline XTE J1946+274 & - & 194539.30 & +272155.0 & B0-1 IV-Ve & 16.8 & 12.5 & 2.0 & 15.8 & 169.2 & 0.33 & $8-10$ \\
\hline KS $1947+300$ & - & 194930.50 & +301224.0 & $\mathrm{~B} 0 \mathrm{Ve}$ & 14.2 & 11.7 & 1.09 & 18.76 & 40.4 & 0.03 & 9.5 \\
\hline EXO $2030+375$ & V2246 Cyg & $2032 \quad 15.20$ & +373815.0 & $\mathrm{~B} 0 \mathrm{e}$ & 19.7 & 12.1 & 3.8 & 41.8 & 46.03 & 0.41 & 5 \\
\hline GRO J2058+42 & - & 205900.00 & +414300.0 & O9.5-B0 IV-Ve & 14.9 & 11.7 & 1.38 & 192 & 110 & - & 9 \\
\hline SAX J2103.5+4545 & - & $2103 \quad 35.71$ & +454505.5 & $\mathrm{~B} 0 \mathrm{Ve}$ & 14.2 & 11.8 & 1.35 & 358.6 & 12.67 & 0.4 & 6.8 \\
\hline $4 \mathrm{U} 2135+57$ & Cep X-4 & 213930.72 & +565910.0 & B1-B2 Ve & 14.2 & 11.8 & 1.3 & 66.3 & - & - & 3.8 \\
\hline SAX J2239.3+6116 & - & 223920.90 & +611626.8 & B0-2 III-Ve & 15.1 & 11.5 & 1.4 & 1247 & 262.6 & - & 4.4 \\
\hline
\end{tabular}


Table 2 Statistics on HMXBs in the Milky Way

\begin{tabular}{lc}
\hline Number of neutron-star X-ray binaries & \\
Number of suspected HMXB & 327 \\
Number of suspected BeXB $^{2}$ & 131 \\
Number of confirmed BeXB & 63 \\
\hline
\end{tabular}

${ }^{1}$ Sources in the Liu et al. (2007) and Liu et al. (2006) catalogs plus update from the 4th IBIS/ISGRI soft gamma-ray survey catalog (Bird et al. 2010)

${ }^{2}$ Sources in the updated on-line version of the Raguzova \& Popov (2005) catalog (http://xray.sai.msu.ru/ raguzova/BeXcat/

${ }^{3}$ Systems with known optical counterpart and verified X-ray behaviour (from Table 1.1)

of Corbet's diagram. Disc-fed SGXBs (squares) show short orbital periods and short spin periods and display an anticorrelation in the $P_{\text {orb }}-P_{\text {spin }}$ diagram. The small orbital separation and evolved companions make Roche lobe overflow the most likely mass transfer mechanism. Wind-fed SGXBs (triangles) show long spin periods and short orbital periods, occupying a more or less flat region in the $P_{\text {orb }}-P_{\text {spin }}$ diagram. Two systems (open triangles) prevent the region from being a horizontal line: OAO1657-41, which might be making a transition to the disc-fed SGXB and 2S $0114+65$, for which the association of the $10^{4} \mathrm{~s}$ pulsations with the spin period of the neutron star remains controversial (Koenigsberger et al. 2006). The spin and orbital periods of BeXB (filled circles) exhibit a clear correlation. The open circle in Fig. 22represents SAX J2103.5+4545, a peculiar system whose X-ray properties are similar to those of wind-fed systems but whose optical/IR emission resemble that of BeXB (Reig et al. 2010a).

The observed correlation in $\mathrm{Be} / \mathrm{X}$ systems is explained in terms of the equilibrium period, defined as the period at which the outer edge of the magnetosphere rotates with the Keplerian velocity (Davidson \& O 1973; Stella et al. 1986; Waters \& van Kerkwijk 1989).

If the the neutron star (and hence the magnetosphere) rotates faster that the equilibrium period, then matter is spun away by the propeller mechanism; only when the spin period is larger than the equilibrium period can matter be accreted on to the neutron star surface. This results in angular momentum transfer to the neutron star, increasing its rotation velocity (decreasing the spin period). The equilibrium period depends mainly on the mass flux (or accretion rate) because it determines the size of the magnetosphere which is assumed to corotate with the neutron star. In turn, the accretion rate depends on the separation of the two components of the binary systems, hence on the orbital period.

The compact object in all confirmed BeXB (Table 1.1) is a neutron star. In fact, many times the neutron star is taken as a defining property of BeXB. It is common to read in the literature that a BeXB consists of a neutron star and a Be star. However, there seems to be no apparent mechanism that would prevent the formation of Be stars with black holes $(\mathrm{BH})$ or white dwarfs (WD). Surprisingly, not a single BeXB is known to host a black-hole companion in our Galaxy, whereas the interpretation of $\gamma$ Cas as a $\mathrm{Be}+\mathrm{WD}$ system still remains very controversial.

Two ideas have been put forward to explain this apparent lack of Be/BH binaries. Zhang et al. (2004) extended the application of the viscous decretion disc model of Okazaki \& Negueruela (2001) to compact companions of arbitrary mass and showed that the most effective Be disc truncation would occur in relatively narrow systems. Using the population synthesis results of Podsiadlowski et al. (2003), which state that binary black holes are most likely to be born in systems with narrow orbits ( $P_{\text {orb }} \lesssim 30$ days), the reason for the lack of these systems can be attributed to the difficulty to detecting them. $\mathrm{Be} / \mathrm{BH}$ binaries are expected to be X-ray transients with very long quiescent states. In contrast, Belczynski \& Ziolkowski (2009) showed that Be/BH binaries do not necessarily have narrow orbits. These strikth rs argued that the predicted ratio of $\mathrm{Be} / \mathrm{NS}$ binaries to $\mathrm{Be} / \mathrm{BH}$ binaries $\left(F_{\mathrm{NS}-\text { to }-\mathrm{BH}} \sim 30-50\right)$ in our Galaxy, based on current population synthesis models and evolutionary scenarios, is consistent with the observations: there are 60 known $\mathrm{Be} / \mathrm{NS}$ binaries, hence one would expect $0-2 \mathrm{Be} / \mathrm{BH}$ binaries, consistent with the observed galactic sample. Both Zhang et al. (2004) and Belczynski \& Ziolkowski (2009) agree in that Be/BH binaries should exist in the Galaxy.

\subsection{Other types of high-mass X-ray binaries}

The traditional picture of two classes of HMXBs, namely SGXB - subdivided into low-luminosity or wind-fed systems and high-luminosity or disc-fed systems - and BeXB - transient and persistent - is giving way to a more complex situation where newly discovered systems may not fit in these categories. 


\subsubsection{Low eccentricity $B e X B$}

There is a group of so far five BeXB (X Per, GS 0834430, KS 1947+300, XTE J1543-568, and 2S 1553-542]), characterised by $P_{\text {orb }} \gtrsim 30 \mathrm{~d}$ and very low eccentricity $(e \lesssim 0.2)$. Their low eccentricity requires that the neutron star received a much lower kick velocity at birth than previously assumed by current evolutionary models (Pfahl et al. 2002).

Most popular models for neutron star kicks involve a momentum impulse delivered around the time of the core collapse that produced the neutron star. These models assume that neutron stars are born with speeds in excess of $100-200 \mathrm{~km} \mathrm{~s}^{-1}$. Such velocities imply a probability close to zero that the post-supernova eccentricity is less than 0.2. Hence, the discovery of these low-eccentricity BeXB with wide orbits was unexpected. Tidal circularizacion is ruled out since this mechanism requires that the star almost fills its Roche lobe and that there be an effective mechanism for damping the tide. Tidal torques should have little effect on the orbit of a HMXB with $P_{\text {orb }}=10$ days, as long as the secondary is not too evolved and the eccentricity is not so large that the tidal interaction is enhanced dramatically at periastron (as it is the case of typical $\mathrm{BeXB})$

Pfahl et al. (2002) developed a phenomenological model that simultaneously accounts for the long-period ( $\lesssim 30$ d), low-eccentricity $(\lesssim 0.2)$ HMXBs and which does not violate any previous notions regarding the numbers and kinematics of other neutron star populations. They propose that a neutron star receives a relatively small kick $\left(\lesssim 50 \mathrm{~km} \mathrm{~s}^{-1}\right)$ if the progenitor, i.e., the core of the initially more massive star in the binary, rotates rapidly. This condition may be met when the progenitor star experienced case $\mathrm{B}_{e}$ or $\mathrm{C}_{e}$ mass transfer in a binary system 2 . If the hydrogen-exhausted core of an initially rapidly rotating massive star is exposed following case Be or Ce mass transfer in a binary, then the core is also likely to be a rapid rotator.

\subsubsection{Obscured sources \& Supergiant fast X-ray transients}

Since the launch of INTEGRAL in October 2002, the situation is also changing among the SGXB group.

\footnotetext{
${ }^{1}$ The optical counterparts of XTE J1543-568 and 2S 1553-542 are not known, hence they do not appear in Table 1.1

${ }^{2}$ In case $\mathrm{B}$, mass transfer occurs during the shell hydrogenburning phase, but prior to central helium ignition, while case $\mathrm{C}$ evolution begins after helium has been depleted in the core. Cases $\mathrm{B}$ and $\mathrm{C}$ are naturally divided into an early case $\left(\mathrm{B}_{e}\right.$ or $\mathrm{C}_{e}$ ), where the envelope of the primary is mostly radiative, and a late case $\left(\mathrm{B}_{l}\right.$ or $\left.\mathrm{C}_{l}\right)$, where the primary has a deep convective envelope.
}

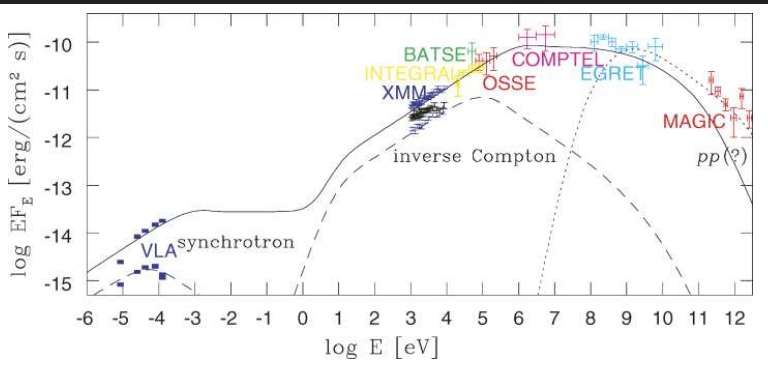

Fig. 3 Spectral energy distribution of LSI +61 303. From Chernyakova et al. (2006)

INTEGRAL has unveiled a population of highly obscured HMXBs with supergiant companions and a new type of source displaying outbursts which are significantly shorter than typical for BeXB and which are characterized by bright flares with a duration of a few hours and peak luminosities of $10^{36}-10^{37} \mathrm{erg}$ $\mathrm{s}^{-1}$. These new systems have been termed as Supergiant fast X-ray Transients (SFXTs, Smith et al. 2006; Nequeruela et al. 2006; Walter \& Zurita-Heras 2007; Negueruela et al. 2008).

Both obscured HMXBs and SFXTs display X-ray and IR spectra typical of SGXBs. In some cases, the X-ray sources are pulsed and orbital parameters typical of persistent SGXBs have been found (e.g., Bodaghee et al. 2006; Zurita Heras et al. 2006). The heavily-absorbed sources had not been detected by previous missions due to high absorption, which renders their spectra very hard. The current understanding is that the entire binary system is surrounded by a dense and absorbing circumstellar material envelope or cocoon, made of cold gas and/or dust (Chaty 2008).

SFXTs differ from SGXBs because they are only detected sporadically, during very brief outbursts (Romano et al. 2010). A promising model to explain SFXTs invokes highly structured (clumpy) stellar winds. The outburst occurs as a result of the accretion of one of the clumps of dense matter from the wind. An alternative model ("Be-type" model) assumes a very elliptical orbit for the binary. In this model outbursts are triggered when the compact object travels through its periastron. Other possibilities imply that SFXTs contain strongly magnetised neutron stars. The outbursts result from the overcoming of centrifugal and magnetic barriers (see Grebenev 2010, and references therein).

\subsection{3 $\gamma$-ray binaries}

$\gamma$-ray binaries are HMXBs that emit most of their radiative output in the $\mathrm{MeV}-\mathrm{TeV}$ range. Currently, only four HMXBs are well-established members of this group of high-energy sources: LS I +61 303, LS 5039, PSR 
B1259-63, and Cygnus X-3, while other two are firm candidates: Cygnus X-1, and HESS J0632+0573. The optical counterpart is either a luminous O-type star (Cygnus X-1, LS 5039), a Be star (LS I +61 303, PSR B1259-63, HESS J0632+057) or a likely Wolf-Rayet star in Cygnus X-3. In addition to the variable $\gamma$-ray emission these systems share in common a resolved radio counterpart with a jet or jet-like structure, multiwavelength orbital modulation and spectral energy distribution (Fig. 31). The wide range of orbital parameters (Paredes 2008) and the non-unique nature of the compact companion (unknown in most systems but with a confirmed neutron star in PSR B1259-63 and a fairly secure black hole in $\mathrm{Cyg} \mathrm{X}-1$ ) represent a challenge for the theoretical modelling of these systems. Two alternative scenarios may explain the variable $\gamma$-ray emission: the microquasar or accretion-powered scenario and the pulsar wind scenario.

All confirmed $\gamma$-ray binaries show a jet or jet-like radio structure, which would indicate the presence of relativistic particles. In black-hole binaries the radio jet can account for their broad-band spectrum, from radio to X-rays (Markoff et al. 2003) as well as for the origin of most of the timing variability (Kylafis et al. 2008). Therefore, it is reasonable to think that the jet may also be the origin of the very high-energy $\gamma$ rays. In the context of relativistic jets, the most efficient gamma-ray mechanism would be inverse Compton scattering, by which relativistic particles collide with stellar and/or synchrotron photons and boost their energies to the VHE range. Two flavors of the microquasar model can be found in the literature depending on whether hadronic or leptonic jet matter dominates the emission at such an energy range. Among leptonic jet models, there are inverse Compton jet emission models in which X-rays and $\gamma$-rays result from synchrotron selfCompton processes (Atoyan \& Aharonian 1999), or in which the seed photons come from external sources, i.e., companion star and/or accretion disk photons Kaufman Bernadó et al. 2002; Georganopoulos et al. 2002). In the hadronic scenario, the gamma-ray emission arises from the decay of neutral pions created in the inelastic collisions between relativistic protons in the jet and either the ions in the stellar wind of the massive companion star (Romero et al. 2003) or nearby high-density regions (i.e. molecular clouds) (Bosch-Ramón et al. 2005).

Alternatively, relativistic particles can be injected in the surrounding medium by the wind from a young

${ }^{3}$ The Be star HD 215227, likely counterpart of the gamma-ray source AGL J2241+4454, has been suggested as a new candidate Wiliams et al. 2010). pulsar. In the pulsar wind scenario, the rotation of a young pulsar provides stable energy to the nonthermal relativistic particles in the shocked pulsar wind material outflowing from the binary companion. As in the microquasar-jet models, the $\gamma$-ray emission can be produced by inverse Compton scattering of the relativistic particles from the pulsar wind on stellar photons (Torres 2010, and references therein). In the pulsar wind scenario the resolved radio emission is not due to a relativistic jet akin to those of microquasars, but arises instead from shocked pulsar wind material outflowing from the binary (Dubus 2006).

\subsection{4 $\gamma$-Cas like objects}

A growing number of early Be stars discovered in Xray surveys exhibit strong $\mathrm{X} /$ optical flux correlations and X-ray luminosities intermediate between those of normal stars and those of most BeXB in quiescence (Lopes de Oliveira et al. 2006). The optical properties are very similar to those of BeXB: $i$ ) the spectral type is always in the range B0-B1 III-Ve, and ii) show $\mathrm{H} \alpha$ equivalent widths stronger than $-20 \AA$. However, they differ from the typical BeXB in their X-ray properties: i) they show harder X-ray spectra that can be best fitted by a thin thermal plasma with $T \sim 10^{8} \mathrm{~K}$, rather than a power law as seen typically in HMXBs, ii) there is no evidence for coherent pulsations in any of these systems but strong variability on time scales as short as $100 \mathrm{~s}$ is usually observed, and iii) they do not exhibit large X-ray outbursts.

The prototype of this group of sources is $\gamma$ Cas. Two models have been put forward to explain this type of system: accretion onto a compact object (most likely a white dwarf) and magnetically heated material between the photosphere of the B star and the inner part of its disc (Robinson et al. 2002). In the magnetic corona model the hard X-rays result from highenergy particles that are emitted due to magnetic reconnection, while the optical variability is due to changes in the density structure of the inner disc as a consequence of turbulence generated by changes in the magnetic field. In support of this scenario there is the fact that X-ray fluxes show random variations with orbital phase, thereby contradicting the binary accretion model, which predicts a substantial modulation. In favour of the binary model is the similarity of the X-ray spectra with those of cataclysmic variables, the rapid variability and large numbers of $\mathrm{Be}+$ white dwarf systems predicted by the theory of evolution of massive binaries.

In the remaining part of this report I shall concentrate on the properties and variability of BeXBÓnly 

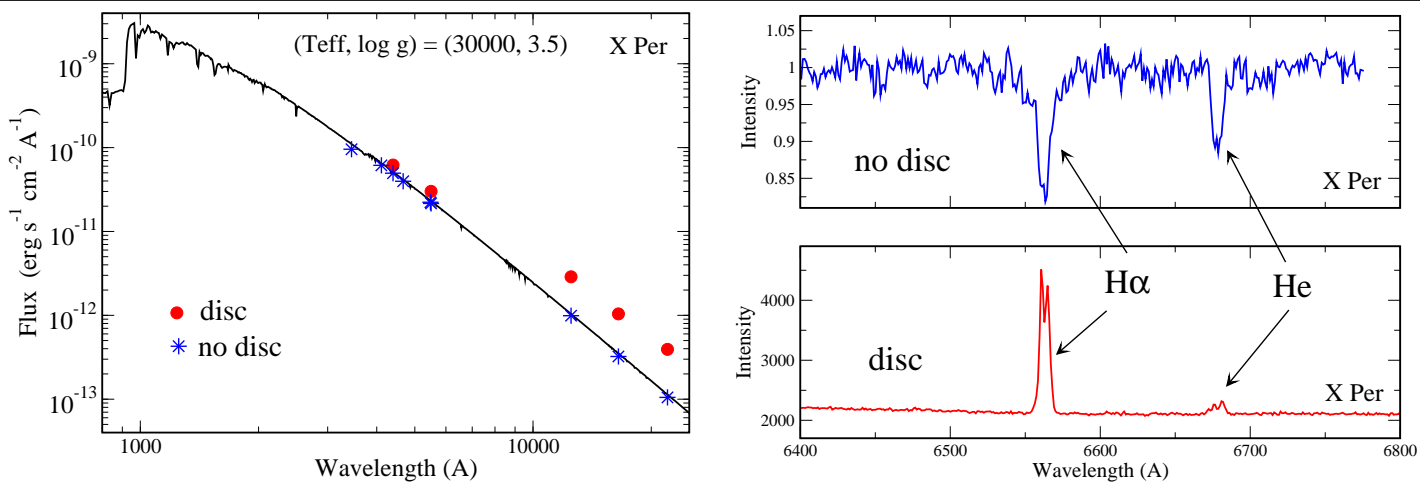

Fig. 4 IR excess and $\mathrm{H} \alpha$ emission are the two main observational characteristics of Be stars.

when for the sake of the discussion a comparison with the behaviour of other type of X-ray binaries may be illustrative, will these other type of binaries be mentioned.

\section{Optical/IR properties of BeXB}

The optical and infrared flux of a BeXB is completely dominated by the Be star companion. While the X-ray emission from BeXB provides information on the physical conditions in the vicinity of the compact object, the optical and infrared emission reveals the physical state of the mass donor component. Since the fuel that powers the X-ray emission, namely matter from a powerful stellar wind or from a equatorially concentrated denser disc, comes from the massive companion, observations of BeXB in the optical/IR are crucial to understand the physical conditions under which the neutron star is accreting.

The two main observational characteristics of Be stars are the emission spectral lines, as opposed to the normal absorption photospheric lines and an excess of IR emission. Both properties, line emission and IR excess, originate from extended circumstellar envelopes of ionised gas surrounding the equator of the B star. They result from free-free and free-bound emission from the disc, i.e. recombination of the optical and UV radiation from the central star (Woolf et al. 1970; Gehrz et al. 1974). The assumption of a common origin for the line emission and IR excess is strongly supported by the correlation between the intensities of continuous IR emission, as measured either by colour indices $(J-M)$, $(J-K)$ or fluxes at a certain wavelength and the intensity of the $\mathrm{H} \alpha$ line, as measured either as equivalent width or fluxes (Dachs \& Wamsteker 1982; Dachs et al. 1988; Neto \& de Freitas Pacheco 1982). By studying the line variability one can obtain important constraints on the geometry (size, shape) and dynamics (velocity and density laws) of the envelope (Hanuschik 1986, 1995; Dachs 1992).

Figure 4 illustrates graphically these two properties for the system X Persei. When an equatorial disc is present the near-IR emission exceeds that predicted by model atmosphere (shown is a Kuruzc model with $T_{\text {eff }}=30000 \mathrm{~K}$ and $\left.\log g=3.5\right)$ and emission lines are prominently seen.

\section{$2.1 \mathrm{H} \alpha$ line profiles}

The $\mathrm{H} \alpha$ line is the prime indicator of the circumstellar disc state. $\mathrm{H} \alpha$ emission lines can be morphologically divided in two classes (Hummel \& Vrancken 1995; Silaj et al. 2010): class 1 are symmetric and includes symmetric double-peak, wine-bottle and shell profiles and class 2 , which are asymmetric and show variability on time scales of years. Each class does not refer to different groups of sources because individual Be stars can change from one to the other. These changes are normally slow (of the order of years to decades in isolated Be stars and months to years in BeXB). Symmetric profiles are believed to be generated in quasi-Keplerian discs (see e.g. Hummel 1994), while asymmetric profiles are associated with distorted density distributions (Hanuschik 1995; Hummel \& Hanuschik 1997).

Most BeXB show asymmetric split $\mathrm{H} \alpha$ profiles. The peaks adopt the names of the relative position of their central wavelengths. In a spectrum with monotonically increasing wavelength values, the left peak is known as the "blue" (or violet) peak, while the right peak is named the "red" peak. V/R variability refers then to the variation of the relative strength of the blue to the red peak. Therefore the $\mathrm{V} / \mathrm{R}$ ratio, defined as the ratio of violet-side to red-side peak intensities above continuum in units of continuum intensity represents a measure of the asymmetry of the line. A more convenient 
quantity is the logarithmic of this ratio, $\log (V / R)$, because in this case, positive values of $\log (V / R)$ correspond to a blue-dominated profile and negative values to a red-dominated line. The top panel of Fig. 5 displays the V/R variability of the BeXB LS I +61 235 (Reig et al. 2000).

$\mathrm{V} / \mathrm{R}$ variability can be explained in terms of a nonaxisymmetrical equatorial disc in which a one-armed perturbation (a zone in the disc with higher density) propagates (Okazaki 1991, 1996, 1997; Papaloizou et al. 1992; Savonije \& Heemskerk 1993). Double-peak symmetric profiles are expected when the high-density part is behind or in front of the star, while asymmetric profiles are seen when the high-density perturbation is on one side of the disc. More precisely, when the highdensity part of the disc perturbation is located on the side of the disc where the rotational motion is directed away from us, we see enhanced red emission $(\mathrm{V}<$ $\mathrm{R}$ ), while when the high-density part is moving toward the observer, blue-dominated profiles $V>R$ are expected (Telting et al. 1994). For systems seen at highinclination angle, the two symmetric cases can be readily distinguished since the central depression between the two symmetric peaks would be more pronounced (reaching or going beyond the continuum, the so-called shell profile), when the perturbation is between the observer and the star. The reason is that shell profiles are thought to be due to a perspective effect, namely, when the line of sight toward the star probes the equatorial disc and self-absorption is produced Rivinius et al. 2006). If the density perturbation revolves around the star in the same direction as the material in the disc (prograde precession), then the $\mathrm{V} / \mathrm{R}$ sequence would be according to Telting et al. (1994): $V=R$ (perturbation behind the star) $\longrightarrow V>R \longrightarrow V=R$ (perturbation in front of the star, shell profile) $\longrightarrow V<R$.

One prediction of the model is that no changes in the slope of the infrared continuum are expected because the $V / R$ variations are not the result of changes in the radial gradient of the circumstellar gas. This is exactly the behaviour that it is found in LS I +61 235 (bottom panel of Fig. (5). While the individual infrared photometric bands changed $(\Delta J \approx \Delta H \approx \Delta K \sim 0.3$ magnitudes) the infrared colours (e.g. $J-K$ ) remained unchanged (Reig et al. 2000).

$\mathrm{V} / \mathrm{R}$ variability is also seen in other lines, like HeI $6678 \AA$. Since helium lines are generated at smaller disc radii than the hydrogen lines Hummel \& Vrancken 1995; Jaschek \& Jaschek 2004), the asymmetry of the HeI line profiles indicates that the internal changes of the disc are global, affecting its entire structure.

$\mathrm{V} / \mathrm{R}$ quasi-periods in BeXB range from 1-5 yr (Table 5) and are shorter than those seen in isolated Be
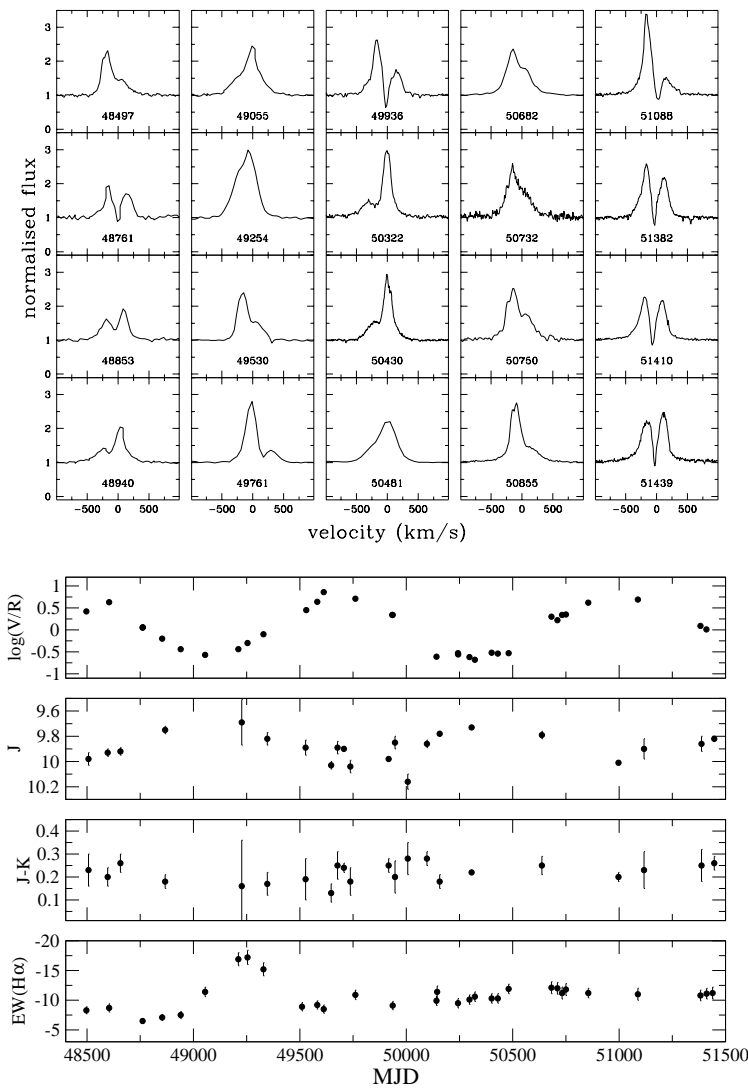

Fig. 5 Top: $\mathrm{V} / \mathrm{R}$ variability observed in the $\mathrm{H} \alpha$ line of LS I +61 235. From Reig et al. (2000). Bottom: Evolution of the $V / R$ ratio, $J$ magnitude, $J-K$ colour and the $\mathrm{H} \alpha$ equivalent width in LS I +61 235. From Reig et al. (2000).

stars, which are found in the range 2-11 years with an average of 7 years (Okazaki 1997).

\section{$2.2 \mathrm{EW}(\mathrm{H} \alpha)$ vs infrared colours}

If the infrared excess observed in Be stars is due to the same processes as those responsible for Balmer line emission, namely absorption and subsequent reemission of the optical and UV light from the underlying star in the circumstellar envelope, then a correlation between IR colours and the strength of the hydrogen lines should be expected. Such correlation has been reported for isolated Be stars (Dachs \& Wamsteker 1982; Neto \& de Freitas Pacheco 1982; Dachs et al. 1988) and BeXB (Coe et al. 1994, 2005, although mixed with isolated Be stars). Figure 6 shows the first such diagram made from BeXB only. It displays the IR colour index $(J-K)_{0}$ as a function of the $\mathrm{H} \alpha$ equivalent width, $\mathrm{EW}(\mathrm{H} \alpha)$, for the sources listed in Table 3 The infrared colours were corrected for interstellar extinction. Only contemporaneous data (when the time difference between the IR and optical observations was less than 


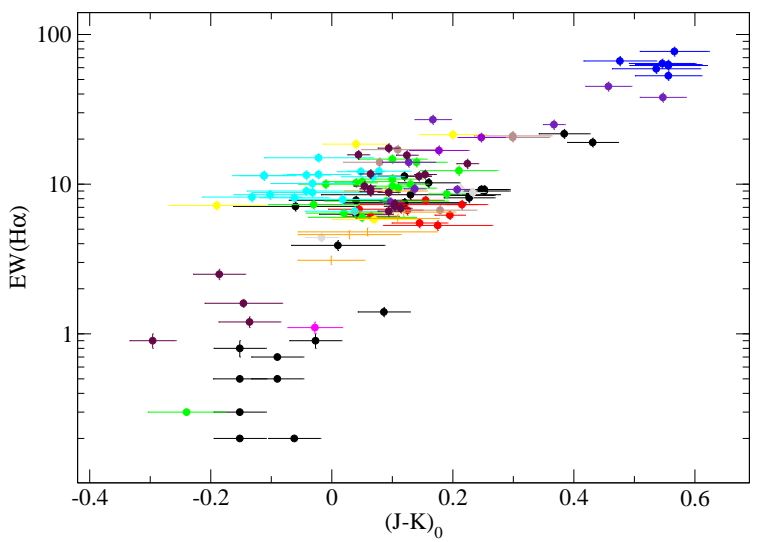

Fig. 6 Correlation between the equivalent width of the $\mathrm{H} \alpha$ line and the infrared colour $J-K$. Different colours represent different systems. This correlation implies a common origin for the infrared excess and the line emission, namely, the circumstellar disc round the Be star.

one month) were used. The second and third columns in Table 3 represent the amplitude of change in the infrared colour $(J-K)$ and the $\mathrm{EW}(\mathrm{H} \alpha)$ over the observed range, whereas Fig. 6 plots the actual values.

In Fig. 6 we have included only those sources for which we are confident that the colour excess $E(B-V)$ is free of circumstellar effects. Due to the surrounding envelope, the use of photometric magnitudes and colours to derive astrophysical parameters, like $E(B-$ $V)$, may be misleading because they are contaminated by disc emission. The effect of the disc is to make the photometric indices to appear redder than a nonemitting B star of the same spectral type. The disc emission makes a maximum contribution to the optical $(B-V)$ colour of a few tenths of a magnitude (Dachs et al. 1988; Howells et al. 2001). The values of $E(B-V)$ used to produce Fig. 6 were obtained from

Table 3 List of BeXB used in Fig. 6 with colour excess and amplitude of change in $(J-K)_{0}$ and $\mathrm{EW}(\mathrm{H} \alpha)$.

\begin{tabular}{lccc}
\hline X-ray source & $\begin{array}{c}E(B-V) \\
(\mathrm{mag})\end{array}$ & $\begin{array}{c}\Delta(J-K)_{0} \Delta E W(\mathrm{mag}) \\
(\AA)\end{array}$ \\
\hline 4U 0115+63 & 1.65 & 0.8 & 20 \\
RX J0146.9+6121 & 0.93 & 0.7 & 10 \\
V 0332+53 & 1.88 & 0.4 & 6 \\
4U 0352+30 & 0.39 & 1.1 & 18 \\
RX J0440.1+4431 & 0.65 & 0.3 & 5 \\
1A 0535+262 & 0.75 & 1.0 & 15 \\
4U 0728-25 & 0.73 & 0.1 & 2 \\
RX J0812.4-3114 & 0.65 & 0.2 & 5 \\
GRO J1008-57 & 1.90 & 1.0 & 15 \\
1A 1118-616 & 0.90 & 1.5 & 25 \\
4U 1145-619 & 0.29 & 2.8 & 40 \\
EXO 2030+375 & 3.8 & 1.1 & 15 \\
SAX J2103.5+4545 & 1.35 & 0.1 & 1 \\
\hline
\end{tabular}

either diffuse interstellar bands, disc-loss episodes or by disentangling the circumstellar and interstellar reddening (see Fabregat \& Torrejón 1998).

Under these conditions, namely, simultaneity of the optical and infrared data and used of the extinctioncorrected $(J-K)$ index, the $E W(H \alpha)-(J-K)_{0}$ becomes a useful tool to estimate the extra reddening caused by the circumstellar disc. Once a value of the $\mathrm{EW}(\mathrm{H} \alpha)$ is known, one can look for the corresponding intrinsic $(J-K)$ in Fig. 6. By comparing this value with that expected according to the spectral type, an estimated of the disc contribution on the index $(J-K)$ can be obtained. The relatively large scatter, however, may limit the usefulness of the diagram.

It should be noticed that although the $\mathrm{H} \alpha$ and infrared emission correlate as expected for a common origin in the disc, the spatial extension and precise localisation in the disc are different. From long-baseline interferometric observations in the $K^{\prime}$ band Gies et al. (2007) found that the angular size of the infrared emission is consistently smaller than that determined for the $\mathrm{H} \alpha$ emission. In other words, the near-IR emission forms closer to the star than does the $\mathrm{H} \alpha$ emission. Furthermore there is good evidence in several cases (Clark et al. 2001; Grundstrom et al. 2007) that an increase in disc brightness occurs first in the near-IR flux excess and later in $\mathrm{H} \alpha$ as expected for an outwardly progressing density enhancement. This difference can be explained if the dependence with disc radius of the $\mathrm{H} \alpha$ optical depth is less steep than that of the infrared optical depth (Gies et al. 2007).

\subsection{Identification of the optical counterparts}

With the improved sensitivities of the currently operational space missions many new X-ray sources are being discovered. About one third of the the $\mathrm{X} / \gamma$-ray sources in the 4th INTEGRAL catalogue (Bird et al. 2010) are thought to be X-ray binaries, of which half are believed to contain early-type companions. An optical identification is necessary to facilitate a complete study of these systems. Without a known counterpart, observations are limited to X-ray energies, and hence our understanding of the structure and dynamics of those systems that remain optically unidentified is incomplete.

If the only available information is that provided by an isolated X-ray detection then potential HMXBs are selected as those exhibiting properties of a magnetised neutron star, namely, X-ray pulsations and/or an absorbed power-law continuum spectrum with an exponential cutoff at 10-30 keV and cyclotron absorption features. Extra information on the nature of the source can be obtained if the long-term X-ray variability is 

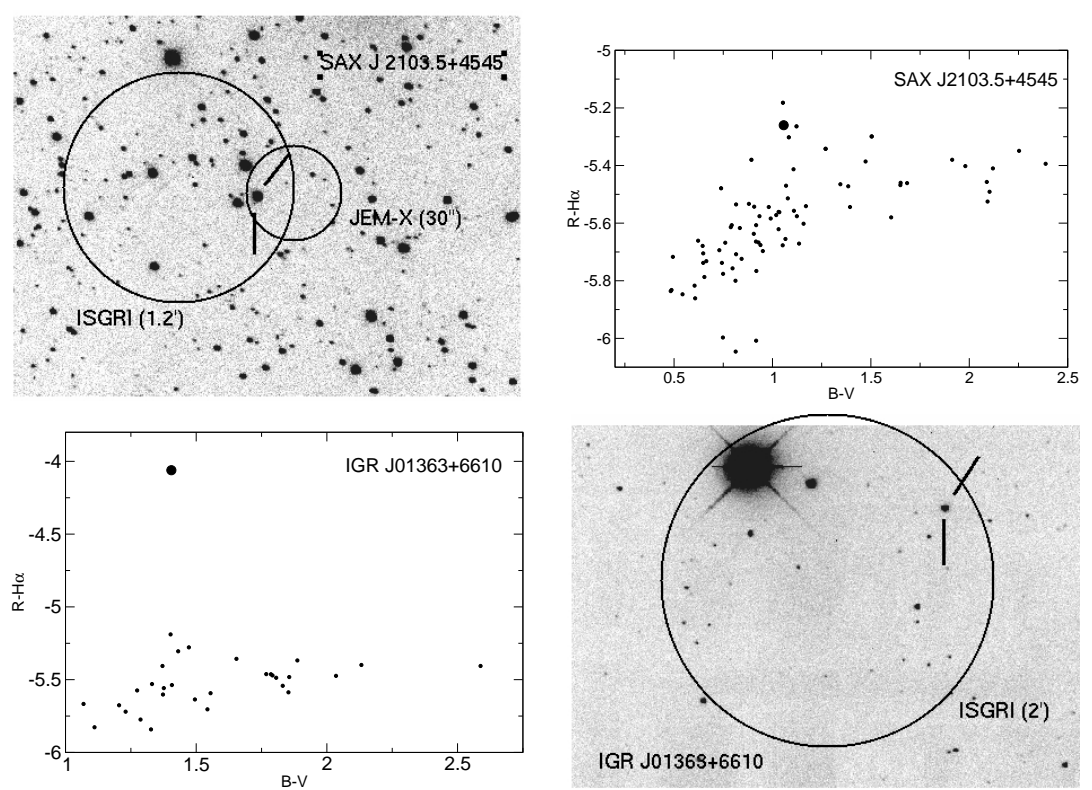

Fig. 7 Colour-colour diagrams and V-band images of the field around SAX J2103.5+4545 (top) and IGR J01363+6610 (bottom). The position of the confirmed optical counterpart in the colour-colour diagram is marked with a filled circle. Good candidates are those that occupy the upper part of the colour-colour diagram and lie inside or very close to the X-ray uncertainty region. For example, the stars that are close to SAX J2103.5+4545 in the colour-colour diagram lie far away from the satellite error circles.

known (information provided by all-sky monitors, like ASM RXTE or BAT SWIFT). The presence of regular and periodic outbursts or unexpected giant outbursts reaching Eddington luminosity at their peaks may indicate the presence of a Be star. Likewise, erratic flaring, i.e., X-ray variability with changes in the $\mathrm{X}$-ray intensity by a factor $3-5$ over a few minutes might indicate the presence of a strong stellar wind from an early-type, probably evolved, companion.

However, a system with X-ray data only will remain in the category of suspected HMXBs until a confirmed optical identification is performed. The most obvious observational features to look for is $\mathrm{H} \alpha$ emission and near-infrared excess emission. While the detection of these two observational features does not guarantee that the source is indeed an OBe type star, it definitely narrows the number of candidates to a handful of sources.

The size of the uncertainty in the location of the $\mathrm{X}$-ray source, the so-called error circle, determines the type of observational technique to use. If the error circle is small (a few arcseconds) then the number of visible stars in the region is expected to be small and it is possible to perform narrow-slit spectroscopic observations and look for early-type, $\mathrm{H} \alpha$ emitting line stars. If the error radius is large ( $\gtrsim 1$ arcminute), then it is likely to include a large number of sources and hence narrow-slit spectroscopy becomes impractical. In this case colourcolour diagrams can be used to identify good candi- dates. Furthermore, uncertainty regions are given to a certain confidence level, hence it is possible that the true optical companion is located close but outside the $\mathrm{X}$-ray error circle.

Photometric detection of Be stars can be performed by selecting colours directly related to the excess emission both in the $\mathrm{H} \alpha$ line ("red" colour) and the continuum ("blue" colour). Observing through a narrow filter centred on the $\mathrm{H} \alpha$ line and a wider filter also containing this line, e.g. Johnson R filter (Reig et al. 2005), Sloan- $r$ Gutiérrez-Soto 2006) would account for the emission line, while Johnson $B-V$ (Reig et al. 2005) or Strömgren $b-y$ (Gutiérrez-Soto 2006) can be used as reddening indicators. The use of Strömgren $b, y$, and narrow-band $\mathrm{H} \alpha$ photometry through $(b-y, y)$ colourmagnitude and $(b-y, y-H \alpha)$ colour-colour diagrams is particularly suitable to identify Be stars in open clusters (Grebel et al. 1992; McSwain \& Gies 2005).

Stars with a moderately or large $\mathrm{H} \alpha$ excess can be distinguished from the rest because they deviate from the general trend and occupy the upper left parts of the diagram. Be star are expected to show bluer colours, i.e., low $(B-V)$ or $(b-y)$, because they are early-type stars (although they normally appear redder than nonemitting B stars due to the circumstellar disc) and also larger (i.e., less negative) $R-\mathrm{H} \alpha$ colours because they show $\mathrm{H} \alpha$ in emission. Once the number of candidates is reduced to a few systems, narrow-slit spectroscopy becomes feasible. The final step is to obtain a spectrum 

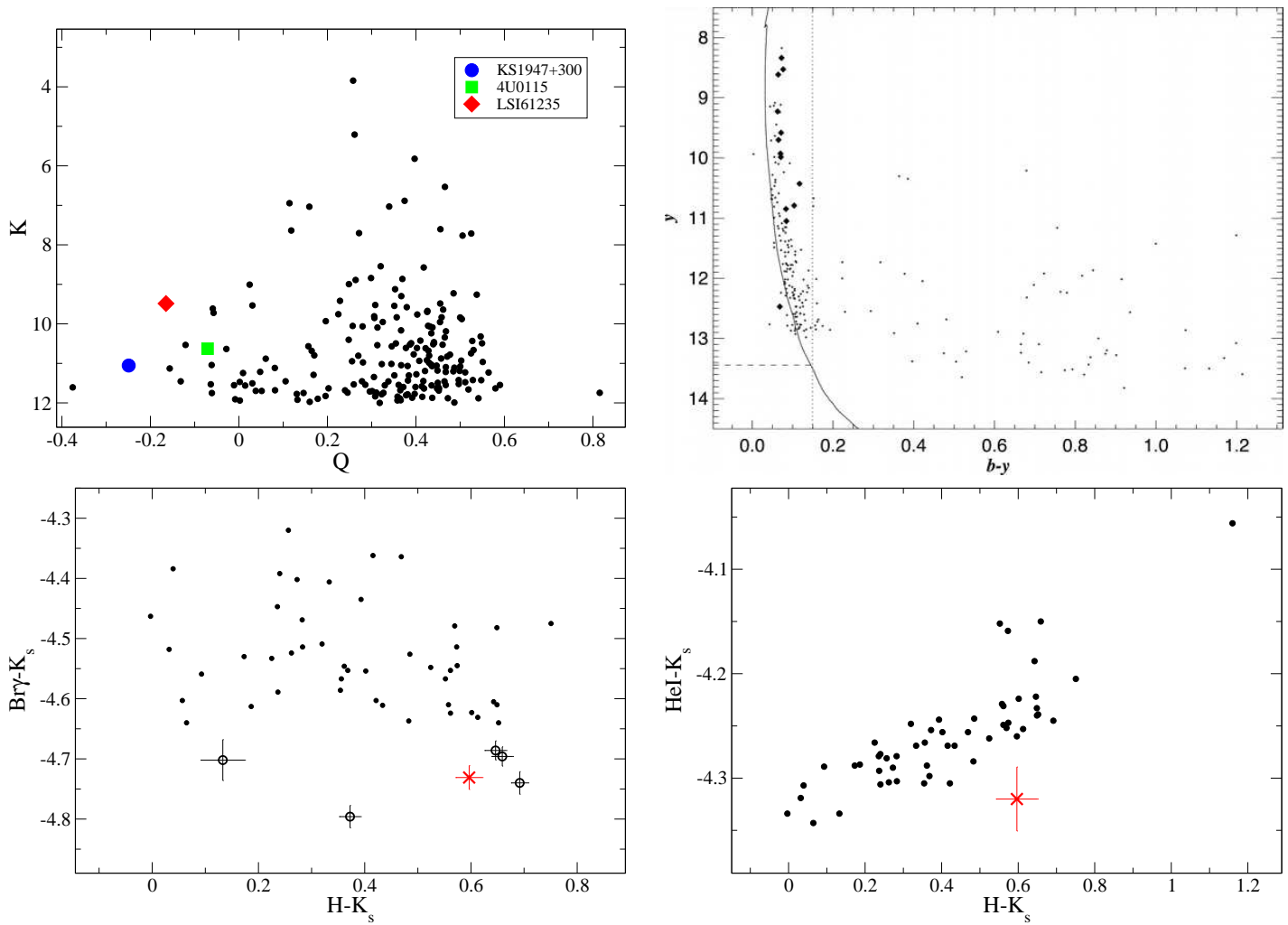

Fig. 8 Top left: $Q-K$ diagram for three well-known systems. BeXB systems tend to occupy the left-most part of this diagram. Top left: $(b-y, y)$ diagram of the open cluster NGC 3766. Be stars (diamonds) are distinguished from all other stars (dots) in the diagram. From McSwain \& Gies (2005). Bottom: IR photometric diagrams of the field around the BeXB 1A 1118-615 (red cross). BeXB candidates (open circles) show up below the sequence of absorption-line stars (black dots).

in the 4000-5000 $\AA$, and perform spectral classification. Figure 7 shows the V-band images and the corresponding $(B-V, R-H \alpha)$ colour-colour diagrams of two recently identified systems

At other wavelengths, similar photometric diagrams have been used. Negueruela \& Schurch (2007) defined the reddening-free quantity $Q=(J-H)-1.70\left(H-K_{\mathrm{S}}\right)$, and created $Q / K_{\mathrm{S}}$ diagrams to separate early type from late type stars. The majority of stars in Galactic fields concentrate around $\mathrm{Q}=0.4-0.5$, corresponding to field $\mathrm{K}$ and $\mathrm{M}$ stars, while early-type stars typically have $Q \simeq 0$. The top left panel in Fig. 8 shows one such diagram with three well-known BeXB.

More recently, Nespoli (2010) built up infrared colour diagrams $\left(\mathrm{Br} \gamma-K_{\mathrm{S}}\right)-\left(\mathrm{H}-K_{\mathrm{S}}\right)$ and $(\mathrm{HeI}-$ $\left.K_{\mathrm{S}}\right)-\left(H-K_{\mathrm{S}}\right)$ to identify emission-line objects. $\operatorname{Br} \gamma$ is the most prominent feature in Be stars in the $K$ band, while He I $20580 \AA$ is found in early type Be stars, up to B2.5 (Clark \& Steele 2000). Emission-line objects show up below the absorption-line stars sequence. Since Be stars present moderate emission line strengths, when compard to other groups (young stellar objects, cataclysmic variables, planetary nebulae), Be star candidates are those below the main sequence but that do not differ more than $\sim 0.3 \mathrm{mag}$ (Fig. 8). The top right diagram in Fig. 8 also shows an example of the use of narrow-band filters to identify Be stars in clusters (from McSwain \& Gies 2005).

\section{X-ray properties}

Tradiationally, BeXB have been considered as transient $\mathrm{X}$-ray binaries harbouring a fast rotating neutron star that orbits a Be stype star in a rather eccentric orbit $(e \gtrsim 0.3)$. Although X-Per was recognized as a BeXB since the beginning of the suggestion of the existence of this type of objects in the early 70's (Moffat et al. 1973), it was the only BeXB showing permanent low level X-ray emission. As the number of BeXB systems began to grow, the X-ray behaviour increased in complexity and new persistent sources were discovered (Reig \& Roche 1999).

Because all BeXB are variable and the detectability of a source depends on the sensitivity of the detector used, the distinction between transient and persistent sources undergoing a large intensity increase is sometimes ambiguous. The term transient is normally used 

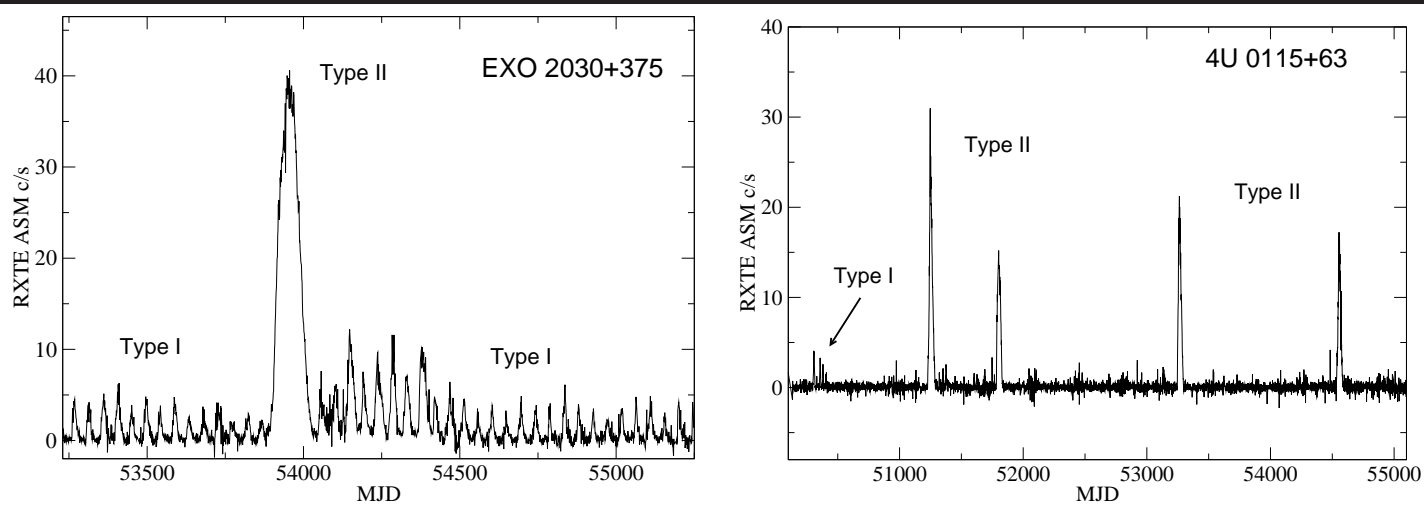

Fig. 9 Long-term light curves of EXO 2030+375 (left) and 4U 0115+63 (right). Although the two systems have been seen to display both type I and type II outbursts, type II outbursts in EXO $2030+375$ are rare whereas type I are almost always present. In $4 \mathrm{U} 0115+63$ the opposite occurs.

when the variability of the source exceeds, at least, two orders of magnitude with respect to the quiescent state, which may correspond to a non-detection state.

\subsection{Persistent versus transient X-ray emission}

Reig \& Roche (1999) proposed the following characteristics differentiating between persistent and transient BeXB. Persistent sources are characterised by

- low X-ray luminosity, $L_{2-20 \mathrm{keV}} \sim 10^{34-35} \mathrm{erg} \mathrm{s}^{-1}$.

- relatively quiet systems showing flat light curves with sporadic and unpredicted increases in intensity by less than an order of magnitude.

- slowly rotating pulsars, $P_{\text {spin }} \gtrsim 200$ seconds.

- absence of, or very weak, iron line at $\sim 6.4 \mathrm{keV}$, indicative of only small amounts of material in the vicinity of the neutron star.

These X-ray properties could be accommodated in systems with wide $\left(P_{\text {orb }} \gtrsim 200\right.$ days $)$ and loweccentric orbits $(e \lesssim 0.2)$. A thermal excess of blackbody type, with high temperature $(k T>1 \mathrm{keV})$ and small emission area $(R<0.5 \mathrm{~km})$, has recently been suggested as another common feature of this type of sources (La Palombara et al. 2009, and references therein). Members of this group are: X Per, RX J0146.9+6121/LS I +61 235, RX J0440.9+4431/BSD 24-491 and RX J1037.5-564/LS 1698.

In contrast, the X-ray behaviour of transient BeXB is characterised by two type of outbursting activity:

- Type I outbursts. These are regular and periodic (or quasiperiodic) outbursts, normally peaking at or close to periastron passage of the neutron star. They are short-lived, i.e., tend to cover a relatively small fraction of the orbital period (typically $0.2-0.3 P_{\text {orb }}$ ). The X-ray flux increases by about one order of magnitude with respect to the pre-outburst state, reaching peak luminosities $L_{x} \leq 10^{37} \mathrm{erg} \mathrm{s}^{-1}$.
- Type II outbursts represent major increases of the X-ray flux, $10^{3}-10^{4}$ times that at quiescence. They reach the Eddington luminosity for a neutron star and become the brightest objects of the X-ray sky. They do not show any preferred orbital phase and last for a large fraction of an orbital period or even for several orbital periods. The formation of an accretion disc during Type II outbursts (Kriss et al. 1983; Motch etal 1991; Havasaki \& Ozakaki 2004; Wilson et al. 2008) may occur. The discovery of quasi-periodic oscillations in some systems (?, and reference therein) would support this scenario. The presence of an accretion disc also helps explain the large and steady spin-up rates seen during the giant outbursts, which are difficult to account for by means of direct accretion.

Figure 9 shows these two types of X-ray variability. The long-term X-ray emission of EXO 2030+375 appears clearly modulated by the orbital period of 46 days just before and after the type II outburst at MJD 53950. 4U 0115+63 has exhibited four type II outburst in the time interval 1996-2009.

Type II outbursts are major events. Since the fuel that powers these outbursts comes from the material in the circumstellar disc, one would expect major changes in the structure of the disc. The disruption should be observable in the parameters that best sample the physical condition in the disc, namely the $\mathrm{H} \alpha$ line parameters (strength and shape) and the IR colours and indices. Figure 10 displays the long-term evolution of the optical/IR and $\mathrm{H} \alpha$ equivalent width of $4 \mathrm{U} 0115+63$. Vertical dotted lines mark the occurrence of type II Xray outbursts. As it can be seen, the large amplitude changes in the optical and IR photometric and spectroscopic parameters after the X-ray outbursts indicate major disruption in the physical conditions of the circumstellar. In fact, the type II outbursts led to the 


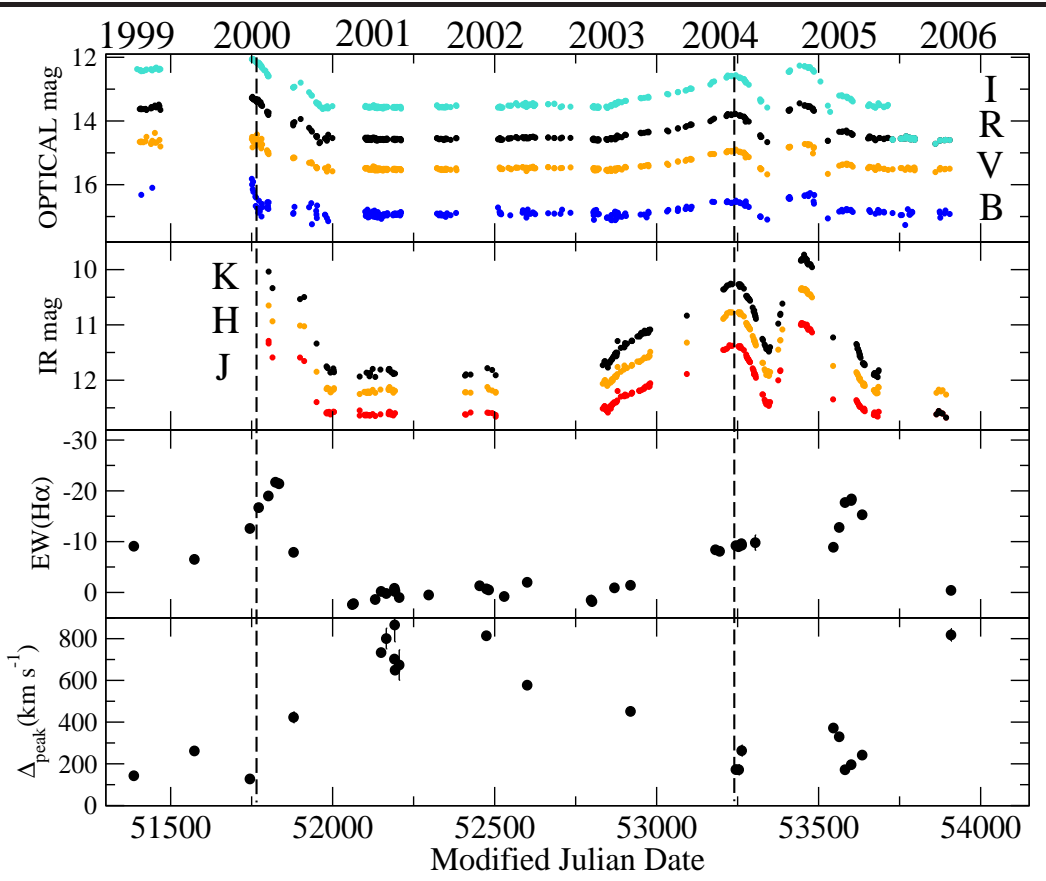

Fig. 10 Long-term evolution of the optical/IR magnitudes and the H $\alpha$ equivalent width and peak separation of doublepeak profiles of $4 \mathrm{U} 0115+63$. Dotted lines mark the occurrence of X-ray outbursts. Adapted from Reig et al. (2007)

complete disappearance of the disc. Evidence for the loss of the disc stems from the $\mathrm{H} \alpha$ equivalent width, which changed sign from negative, indicating that the spectral line was in emission before the outburst to positive, that is absorption, after the outburst.

However, type II outbursts are not always followed by a disc-loss phase. Figure 11 shows the case of $1 \mathrm{~A} 0535+262$ and KS $1947+300$. In the case of $1 \mathrm{~A}$ $0535+262$, the $\mathrm{H} \alpha$ equivalent width decreased significantly after the X-ray outburst, indicating a major disruption in the structure of the disc, but the $\mathrm{H} \alpha$ equivalent width remained negative. The case of KS $1947+300$ is hard to explain. Apparently, the X-ray outbursts produced no effect on the disc. The $\mathrm{H} \alpha$ equivalent width did not change significantly after the outburst.

\subsection{Periodic variability: X-ray pulsations}

Observations of X-ray binaries show considerable variability on a wide range of time scales in all wavelengths. In LMXB, in the so-called millisecond pulsars, the Xray periodic variability goes down to less than a millisecond. In BeXB the shortest timescales are of the order of few second 4 , and correspond to the X-ray pulsations.

${ }^{4}$ There are at least two systems, A0535-66 and SAX J0635+0533, with B-type companions that exhibit millisecond pulsations. However, they are thought to be rotation-powered pulsars.
The detection of coherent pulsations from an accreting X-ray source provides one of the strongest evidence that the compact object is a neutron star. The pulses of high-energy radiation are due to a misalignment of the neutron star's rotation axis and its magnetic axis. Gas is accreted from the stellar companion and is channeled by the neutron star's magnetic field onto the magnetic poles producing two or more localized X-ray hot spots. These hot spots move into and out of view as the neutron star spins, giving rise to regular X-ray pulses.

Almost all BeXB show X-ray pulsations. The pulsation period of an X-ray pulsar with a Be companion can be as short as a few seconds (4U 0115+63) and as long as few tens of minutes (LS I +61 235). The longest periods in main-sequence HMXBs are those of $4 \mathrm{U} 2206+54$ with $1.54 \mathrm{hr}$ (Reig et al. 2009) and IGR J01583+6713 with $5.47 \mathrm{hr}$ (Wang 2009). However, these two sources are somehow peculiar and cannot be considered as canonical BeXB. Although $4 \mathrm{U}$ $2206+54$ exhibits a double-peaked emission $\mathrm{H} \alpha$ profile and the optical companion is an O9.5 main-sequence star, its optical spectrum is complex and far from that of a "typical" Be star (Blav et al. 2006). The 5.47hr period of IGR J01583+6713 needs confirmation as a shorter (469 s) periodicity has also been suggested (Kaur et al. 2008). Among HMXBs with a supergiant companion, 2S 0114+65 shows the longest spin period with $2.78 \mathrm{hr}$ (Finley et al. 1994). The only BeXB for which no pulsations have been detected is LS I +61 

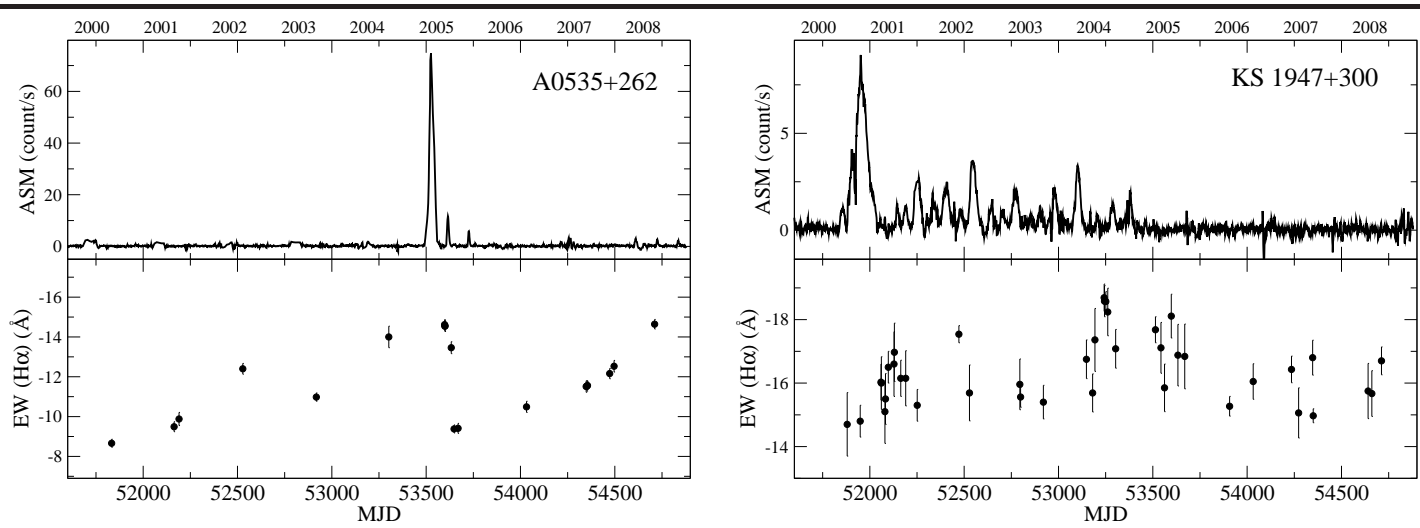

Fig. 11 Long-term evolution of the X-ray intensity (1.3-12.1 keV) and the H $\alpha$ equivalent of $1 \mathrm{~A} 0535+262$ (left) and KS $1947+300$ (right).

$303 / 2 \mathrm{E} 0236.6+6101$. The nature of this system is under debate. The lack of pulsations can be explained if it contains a black hole, that is, if LS I +61 303 is a microquasar. Radial velocity studies do not completely rule out this possibility (Casares et al. 2005a). On the other hand, radio observations favour the presence of a neutron star. However, in this case LS I +61 303 would contain a rotation-powered pulsar with nebula and not an accretion-powered pulsar (Dhawan et al. 2006, see also Sect. 1.2.3).

During major outbursts, transient accretion-powered pulsars show spin-up episodes, with the spin-up rate increasing with accretion luminosity (Fig. 12). This is consistent with the simple model of disc magnetosphere interaction (Ghosh \& Lamb 1979), in which, as the accretion rate increases, the rate of angular momentum transfer from the accretion flow to the neutron star increases. At the limit of very low mass accretion rate, the neutron stars are expected to spin down, because the magnetic field lines that couple to the outer, slower accretion flow remove spin angular momentum from the neutron star.

Spin periods in the range 1-1000 s can be explained according to the current understanding of the neutron star's spin evolution in a close binary system (Davies et al. 1979; Davies \& Pringle 1981; Zhang et al. 2004; Dai et al. 2006). Longer periods are, however, difficult to accommodate in the standard theory. The spin evolution of a neutron star in a binary system is divided into three basic phases or states. Each state is characterised by a different energy release mechanism and corresponds to a different evolutionary stage of the neutron star. These phases are known as the pulsar (or ejector) phase, the propeller phase and the accretion phase. To explain the existence of long-period pulsars ( $P_{\text {spin }} \gtrsim 1000 \mathrm{~s}$ ), a subphase of the propeller state needs to be invoked. This subphase is called the subsonic propeller phase (Davies \& Pringle 1981; Ikhsanov 2001a, b, 2007).

After a supernova explosion a neutron star is formed as a rapidly rotating radio pulsar. The spin period at birth is of the order of a fraction of a second and its magnetic field of the order of $10^{13} \mathrm{G}$. In this phase, the neutron star radiates at the expense of its rotational energy.

The condition for the transition of a slowing-down neutron star from the ejector phase into the supersonic propeller phase occurs when the pressure of the relativistic wind ejected by the neutron star (magnetic pressure) is balanced by the pressure of the plasma surrounding the neutron star (ram pressure) at the gravitational capture or accretion radius $r_{\text {acc }}=2 G M_{x} / v_{w}^{2}$, where $M_{x}$ is the neutron star mass and $v_{w}$ is the stellar wind velocity. The plasma will tend to accrete onto the neutron star under the action of gravitation. The electromagnetic field, however, will obstruct this process, and the accreting matter will come to a stop at the magnetospheric boundary. The accreted material cannot penetrate any further because the drag exerted by the magnetic field is super-Keplerian. The in-falling matter is accelerated outward, taking away the angular momentum of the neutron star. Thus the spin-down of the neutron star continues until the centrifugal and gravity forces acting on a particle that corotates with the neutron star balance. This occurs at the so-called equilibrium period.

If the neutron star spin period is larger than the equilibrium period, then the centrifugal barrier ceases to be effective and plasma that penetrates into the magnetosphere is able to reach the neutron star surface, moving along the magnetic field lines in the direction of the magnetic poles.

Since the material accreted carries substantial angular momentum, the neutron star will experience strong 
spin-up. Therefore, in principle, the equilibrium period given by

$P_{\mathrm{eq}} \approx 23 \mu_{30}^{6 / 7} \dot{M}_{15}^{-3 / 7}\left(\frac{M_{x}}{\mathrm{M}_{\odot}}\right)^{-5 / 7} \mathrm{~s}$

represents the maximum period for a given mass accretion rate. $\mu_{30}$ and $\dot{M}_{15}$ are the magnetic moment and mass accretion rate in units of $10^{30} \mathrm{G} \mathrm{cm}^{3}$ and $10^{15} \mathrm{~g}$ $\mathrm{s}^{-1}$, respectively.

$P_{\text {eq }} \sim 10^{3} \mathrm{~s}$ can be achieved if the magnetic field strength in units of $10^{12} \mathrm{G}, B_{12}>100$ or if $\dot{M}_{15}<$ $10^{-3}$. None of these conditions is supported by the observations. The condition of supercritical magnetic fields would imply that the neutron star is a magnetar. However, the detection of cyclotron lines in the range 10-100 keV indicates that the magnetic field strength is of the order a few times $10^{12} \mathrm{G}\left(B_{12} \sim 1-6\right)$. The low mass accretion rate condition would imply an X-ray luminosity two to three orders of magnitude lower than observed.

In order to explain spin periods longer than $\sim 10^{3}$ $\mathrm{s}$, another phase in the spin-period evolution has to be considered. This phase is called subsonic propeller phase and occurs prior to the accretion-powered phase (Ikhsanov 2001a, b, 2007).

We indicated above that if $P_{\text {spin }}>P_{\text {eq }}$ then matter can be accreted onto the neutron star surface. However, this is actually not always true. Davies \& Pringle (1981) showed that the star's magnetosphere during the propeller spin-down epoch is surrounded by a hot, spherical quasi-static envelope. This envelope extends from the magnetospheric radius $r_{m}=$ $\left(\mu^{2} / \dot{M} \sqrt{2 G M_{x}}\right)^{2 / 7}$ up to the accretion radius $r_{\text {acc }}=$ $2 G M_{x} / v_{w}^{2}$. The interaction between the magnetosphere and the envelope leads to the deceleration of the rotation rate of the neutron star: the rotational energy loss by the star is convected up through the envelope by the turbulent motions and lost through its outer boundary. The neutron star remains in the propeller state as long as the energy input to the envelope due to the propeller action by the star dominates the radiative losses from the envelope plasma.

Steady accretion under the condition $P_{\text {spin }}>P_{\text {eq }}$ can be realized only if the cooling of the envelope plasma (due to radiation and convective motions) dominates the energy input. The subsonic propeller phase corresponds to the phase when this cooling occurs.

The condition for accretion then translates to $P_{\text {spin }}>$ $P_{\mathrm{br}}$, where $P_{\mathrm{br}}$ is the so-called break period, which is given by (Ikhsanov 2007)

$$
\begin{aligned}
& P_{\mathrm{br}} \approx 7 \times 10^{-4} \mu^{16 / 21} M_{x}^{-4 / 21} \dot{M}^{-5 / 7} \\
& P_{\mathrm{br}} \approx 442 \mu_{30}^{16 / 21} \dot{M}_{15}^{-5 / 7}\left(\frac{M_{x}}{\mathrm{M}_{\odot}}\right)^{-4 / 21} \mathrm{~s}
\end{aligned}
$$

While $P_{\text {spin }}<P_{\mathrm{br}}$ the temperature of the envelope remains higher than the free-fall temperature $\left(T_{\mathrm{ff}}\left(r_{m}\right)=\right.$ $G M_{x} m_{p} / k r_{m}$, where $m_{p}$ is the proton mass and $k$ the Boltzmann constant) and no accretion is possible.
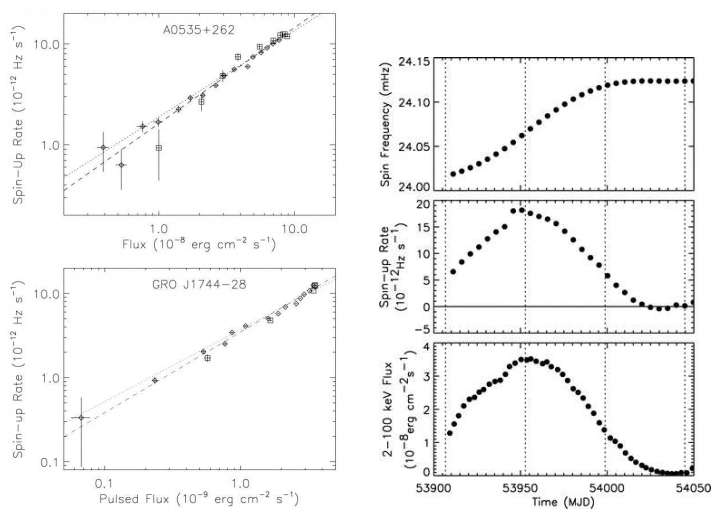

Fig. 12 Observed relationships between flux and pulsar spin-up rate Left: A $0535+262$ during the 1994 giant and GRO J1744+28 during the 1995-1996 outburst (Bildsten et al. 1997). Right: EXO 2030+375 during the 2006 giant outburst (Wilson et al. 2008)

\subsection{Aperiodic variability}

Aperiodic variability refers to the variability caused by processes that do not repeat themselves in a periodic way (van der Klis 1995, 2006). Pulsations, eclipses, outbursts are excluded from the analysis of aperiodic variability studies. In contrast, flickering, irregular flaring, fluctuations are the subject of study. The main tool used for studying the aperiodic variability is the Fourier power spectrum, or a Power Spectral Density (PSD), of the count-rate time series. A number of variability components, or power-spectral components, together make up the power spectrum. An aperiodic component by definition covers several frequency resolution elements. Broad features are called noise and narrow features are called quasi-periodic oscillations (QPOs). The so-called $Q$-factor determines whether a noise component deserves the name QPO. If the $Q$-factor, defined as the ratio between the characteristic frequency of the component over its width, $Q=\nu / F W H M$, is larger than 2 , then the noise component is considered to be narrow enough to be called a QPO.

Aperiodic variability studies are normally carried out in correlation with the position of the source in the 

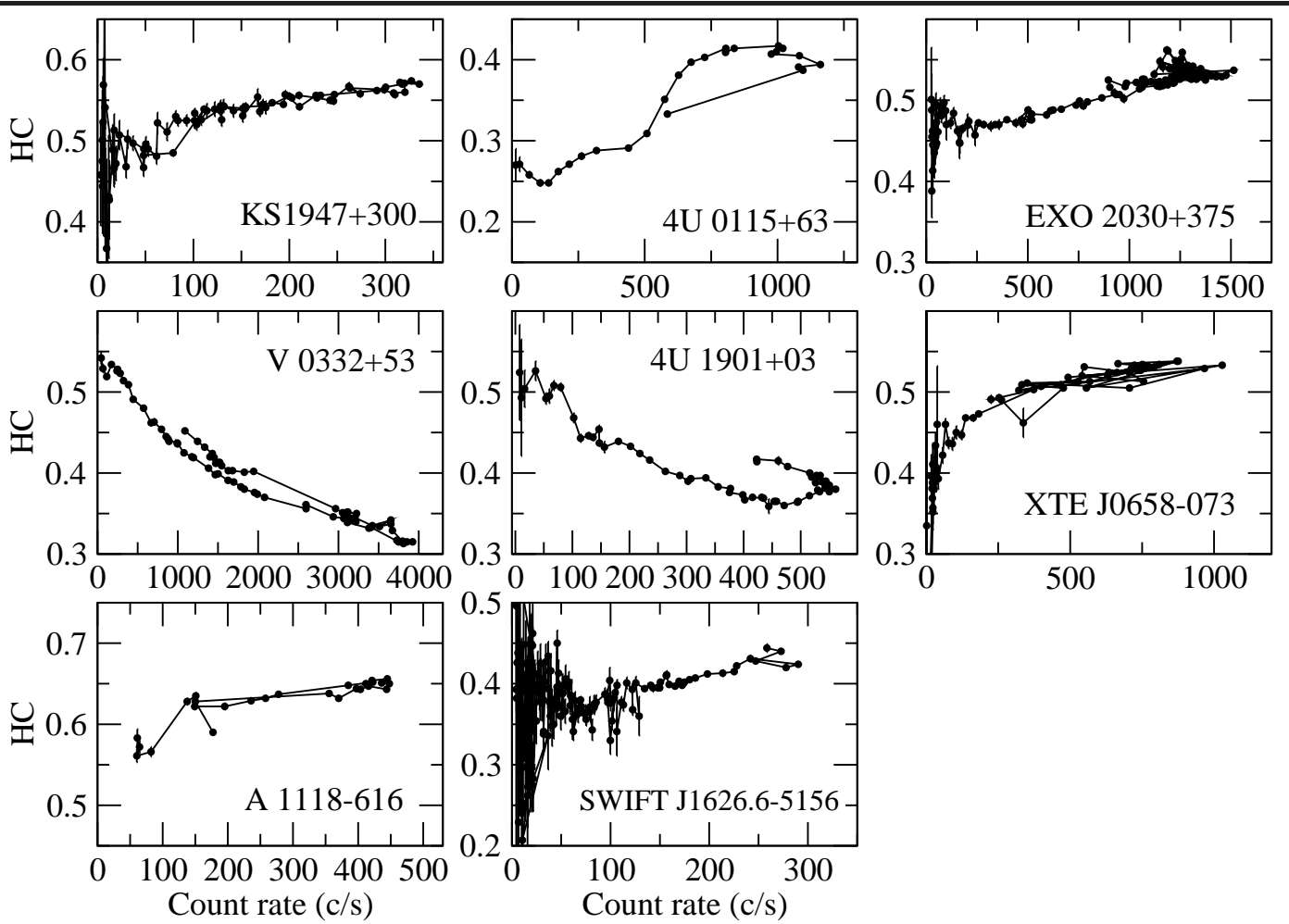

Fig. 13 Hardness-intensity diagrams traced by various BeXB during giant (type II) outbursts observed by $R X T E$. The hard colour, $\mathrm{HC}$, is the ratio between the count rate in the energy range $15-30 \mathrm{keV}$ over $10-15 \mathrm{keV}$.

colour-colour diagram and/or hardness-intensity diagram. An X-ray color is a 'hardness' ratio between the photon counts in two broad bands. A colour-colour diagram (CD) is the plot of one color vs. another calculated in different energy bands. The higher energy range color is called hard colour, while the lower energy range is the soft colour. A hardness-intensity diagram (HID) is instead the plot of a colour vs. the count-rate in some broad spectral range. Figure 13 shows the HID of eight BeXB during type II outbursts. The CD/HID are very useful tools to investigate the spectral variations of an X-ray source because they are model- and instrument-independent and reflect the intrinsic properties of the system.

Together with the functional form of the variability components ("noise"), CD and HID have revolutionised our understanding of X-ray binaries, unveiling a whole new phenomenology on the spectral and timing properties of X-ray binaries by introducing the notion of source states. A state is defined by the appearance of a spectral (i.e. power-law, blackbody) or variability component (i.e. Lorentzian) associated with a particular and well-defined position of the source in the CD/HID. A transition between states takes place when the relative strength of the spectral or variability components varies and the source motion in the $\mathrm{CD} / \mathrm{HID}$ changes direction (van der Klis 2006).
In LMXBs and BHBs the aperiodic variability is thought to originate in the irregular nature of the inner accretion flow, as an accretion disc is assumed to exist in this type of systems. In HMXBs the details of how the magnetic field affects the accretion flow are not fully understood.

While there are numerous references in the literature on the application of CD/HID analysis on LMXBs and BHBs, very little work of this type has been done on HMXBs (Belloni \& Hasinger 1990). Recently, Reig (2008) performed the first systematic analysis of the spectral and rapid aperiodic variability of Be/X-ray binaries during type II outbursts. He found similarities and differences with respect to the behaviour of LMXBs and BHBs.

The similarities include: i) existence of spectral branches in the CD/HID. At high and intermediate flux the sources move along a diagonal branch (DB), while at very low flux the soft colour decreases while the hard colour remains constant defining a horizontal branch (HB); ii) the source does not jump through the diagram but moves smoothly, i.e. without jumps, following the pattern; iii) the broad noise components in the $\mathrm{HB}$ are more variable (in terms of fractional $\mathrm{rms}$ ) than in the DB; $i v$ ) the power spectra of HMXBs can be fitted acceptably with a sum of a small number (2-4) 
of Lorentzian functions (Fig. 14). All sources present peaked noise in certain spectral states.

While the differences are: i) presence of hysteresis patterns in the CD/HID that might be a manifestation of the magnetic field (through cyclotron resonant scattering features); ii) slower motion along the spectral branches, hours to days in LMXBs, days to weeks in HMXBs; iii) the characteristic time scales implied by the noise components are about one order of magnitude longer in HMXBs. There are about 14 HMXBs pulsars that show QPOs in their power spectra (James et al. 2010). The frequencies of these QPOs range from 1$200 \mathrm{mHz}$. In contrast, in LMXBs and BHBs QPO range $1-1000 \mathrm{~Hz} ; i v$ ) the so-called $L_{S}$ noise, whose central frequency coincides with the frequency of the fundamental peak of the pulse period and suggests a strong coupling between the periodic and aperiodic noise components. This coupling implies that the instabilities in the accretion flow that give rise to the aperiodic variability must travel all the way down to the neutron star surface (Lazzati \& Stella 1997; Burderi et al. 1997), and $v$ ) no apparent correlation between the power spectral parameters (characteristic frequencies, $r m s$ ) and mass accretion rate.

\section{Circumstellar disc}

\subsection{Disc formation}

Be stars are fast rotators. They have, on average, larger observed rotational velocities than B stars as a group (Slettebak 1982). The determination of the rotational velocity is believed to be a crucial parameter in the formation of the circumstellar disc. A rotational velocity close to the break-up or critical velocity (i.e. the velocity at which centrifugal forces balance Newtonian gravity) reduces the effective equatorial gravity to the extent that weak processes such as, gas pressure and/or non-radial pulsations may trigger the ejection of photospheric matter with sufficient energy and angular momentum to make it spin up into a Keplerian disc.

At present, there is no consensus on how close to critical velocity Be stars rotate, nor on how Be stars reached such high spinning rates. Observations suggest that a large fraction of Be stars rotate at $70-80 \%$ of the critical value (Slettebak 1982; Porter 1996; Yudin 2001). However, Townsend et al. (2004) claimed that if gravity darkening is taken into account then most or all Be stars may be rotating very nearly at critical velocity. Whether the rotational velocities of Be stars reflect the initial distribution of angular momentum or they have been spun up as a result of binary evolution is still a matter of debate. There is growing evidence that supports the idea that some Be stars were spun up by mass transfer in interacting binaries (Gies 2000). The former mass donors are now found as neutron stars (i.e. BeXB) in some cases and as a small hot subdwarfs stripped of its outer envelope (Phi Per, 59 Cyg, FY CMa) in other cases (Gies et al. 1998; Rivinius et al. 2004; Maintz et al. 2005; Peters et al. 2008).

The projected rotational velocities are determined by measuring the width of certain spectral lines (see e.g. Steele et al. 1999), as it is generally assumed that the width of the spectral lines and the rotational velocity are linearly related. This is true even with the inclusion of limb and intrinsic darkening, but if gravitational darkening is taken into account, then for stars rotating above $\sim 80 \%$ of critical speeds the linear relationship is no longer applicable. Any increase in the rotational velocity is accompanied by almost no change in the line width (Collins \& Truax 1995; Cohen et al. 2005). As a result, the observational parameter $v \sin i$ may systematically underestimate the true projected equatorial rotation velocity (Townsend et al. 2004). In contrast, Cranmer (2005) found that this may be true for the late-type Be stars (i.e., spectral types B3 and later), but early-type Be stars do seem to be consistent with a range of intrinsic rotation speeds between $\sim 40 \%$ and $100 \%$ critical. For stars rotating sufficiently below the critical velocity, any physical model for the origin of Be-star discs requires a significant increase in angular momentum above the photosphere.

While the origin of the gas in these discs is agreed to be material ejected from the stellar photosphere, the precise mechanism triggering this mass ejection remains elusive. One of the first models that tried to explain the formation of circumstellar discs is the wind-compressed model (Biorkman \& Cassinelli 1993). The fundamental idea behind this model is that in a rotating wind, the material in the wind tends to orbit the star during the time it is accelerated outward. If the outward acceleration is small compared to the rotation, then the radiation-driven wind flows toward the equator. Complimentary streamlines from opposite hemispheres would intercept each other at the equatorial plane giving rise to a pair of shocks above and below the equator and eventually the disc. Note that the disc is not a result of enhanced equatorial mass-loss. A major problem of this model arises when non-radial components are included in the radiation force, as these components lead to an effective suppression of the equatorward flow needed to compress the wind (Owocki et al. 1996). In addition, this model was unable to reproduce the IR excess of Be stars (Porter 1997).

The magnetically torqued disc model (Brown et al. 1998) extends the ideas of the wind compressed disc 


\section{$4 \mathrm{U} 0115+63$}
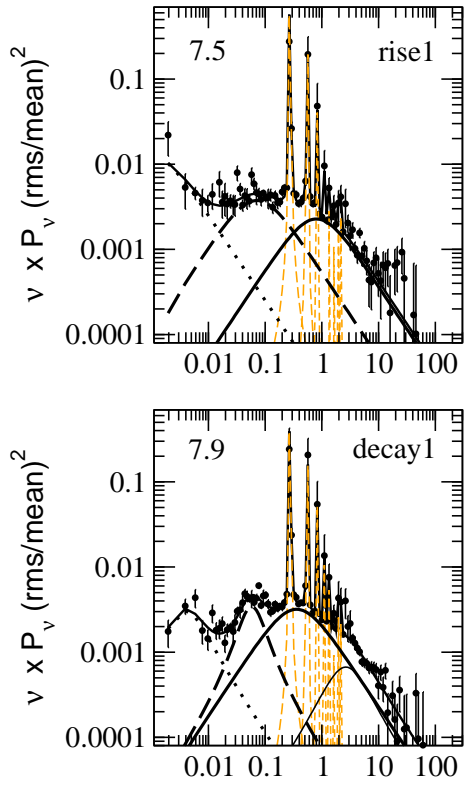
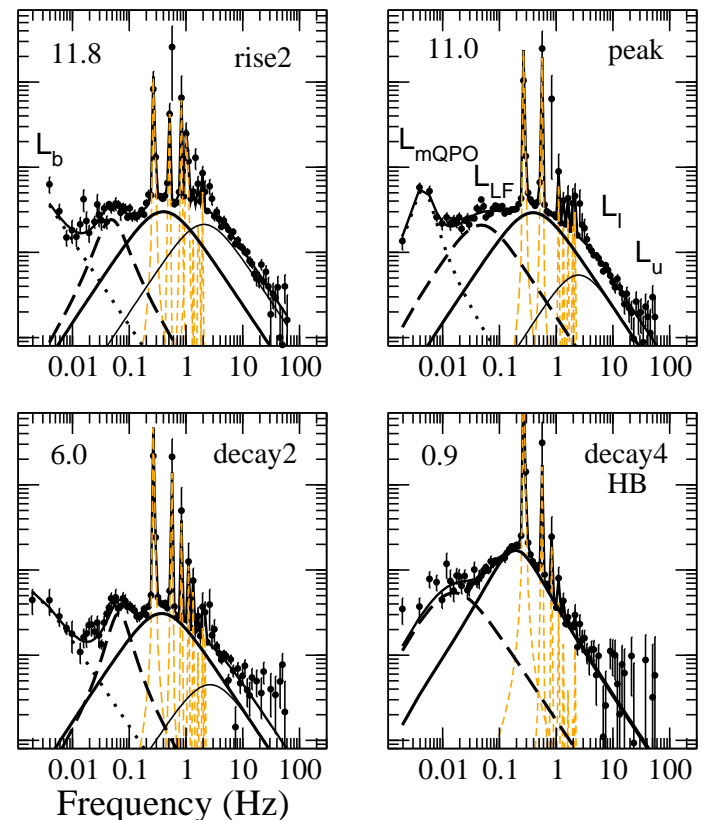

Fig. 14 Power spectra of $4 \mathrm{U} 0115+63$ at various stages of the 2004 giant outburst. The number at the top left of each panel is the X-ray luminosity in units of $10^{37} \mathrm{erg} \mathrm{s}^{-1}$. From Reig (2008).

model by adding magnetic steering and torquing up of the angular momentum of the wind. The up-flowing wind from near the star is magnetically channeled back to the equator. This obviates the Owocki et al. (1996) objections such as the driving of a polar flow by the nonradial radiation field. The magnetically torqued disc model requires a dipole-like magnetic field to channel the flow of wind material into a disc region along the equatorial plane.

An alternative scenario that has been put forward to elevate disc matter to orbits well above the stellar surface and which is increasing in popularity is non-radial pulsations (Cranmer 2009, and references therein). Non-radial pulsations may give rise to outwardly propagating circumstellar waves, which inject enough angular momentum into the upper atmosphere to spin up a Keplerian disc. The main uncertainty in this model is the connection between pulsations and resonant waves since pulsations themselves are evanescent in the stellar photosphere. However, once the resonant waves have been formed, they grow in amplitude with increasing height, begin to propagate upwards, and steepen into shocks. The resulting dissipation would create substantial wave pressure that both increases the atmospheric scale height and transports angular momentum upwards.

Once the disc is formed, the viscous decretion disc model (Lee et al. 1991; Okazaki 2001) stands up as a very promising model that explains many of the observed features in discs around Be stars. In this model, matter supplied from the equatorial surface of the star gradually drifts outward by the viscous torque and forms the disc. Decretion discs operate in a similar fashion to accretion discs, except that the sign of $\dot{M}$ (mass decretion/accretion rate) is opposite. Viscosity is treated following the Shakura-Sunyaev's $\alpha$ prescription. The outflow is highly subsonic in the inner part of the disc and close to Keplerian. Although aspects of the modeling (e.g., the supply of matter with sufficient angular momentum to the inner edge of the disc or the interaction of the stellar radiation with the disc matter) are not fully solved yet, the model is able to explain the very low outflow velocities (the observed upper limits are, at most, a few $\mathrm{km} \mathrm{s}^{-1}$ ) and also the $\mathrm{V} / \mathrm{R}$ variability observed in Be stars.

\subsection{Disc-loss episodes}

The use of photometric calibration for the determination of the spectral type and luminosity class in a Be star is not as straightforward as in a non-emission-line B-type star owing to the presence of the surrounding envelope, which distorts the characteristic photospheric spectrum. Be stars appear redder than the non-emission B stars, owing to the additional reddening caused by the hydrogen free-bound and free-free processes in the circumstellar envelope.

Thus, in a Be star, one has to correct for both circumstellar and interstellar reddening before any calibration can be used. There is no easy way to decouple 
Table 4 Rotational velocity of Be/X-ray binaries

\begin{tabular}{lllccc}
\hline $\begin{array}{l}\text { X-ray } \\
\text { source }\end{array}$ & $\begin{array}{l}\text { Optical } \\
\text { counterpart }\end{array}$ & $\begin{array}{l}\text { Spectral } \\
\text { type }\end{array}$ & $\begin{array}{c}\text { Inclination } \\
\text { angle }\left(^{\circ}\right)\end{array}$ & $\begin{array}{c}v \text { sin } i \\
\left(\mathrm{~km} \mathrm{~s}^{-1}\right)\end{array}$ & Reference \\
\hline 4U 0115+634 & V635 Cas & B0.2V & 43 & $300 \pm 50$ & 1 \\
RX J0146.9+6121 & LS I +61 235 & B1III-V & - & $200 \pm 30$ & 2 \\
V 0332+53 & BQ Cam & O8-9V & $<10$ & $<150$ & 3 \\
X-Per & HD 24534 & O9.5III & $23-30$ & $215 \pm 10$ & 4,5 \\
RX J0440.9+4431 & LS V +44 17 & B1III-V & & $235 \pm 15$ & 6 \\
1A 0535+262 & HD 245770 & O9.7III & $28-35$ & $225 \pm 10$ & 7,8 \\
RX J0812.4-3114 & LS 992 & B0.5III & - & $240 \pm 20$ & 9 \\
1A 1118-615 & Hen 3-640 & O9.5IV & - & 300 & 10 \\
4U 1145-619 & V801 Cen & B0.2III & $<45$ & $250 \pm 30,290$ & 11,12 \\
4U 1258-61 & V850 Cen & B2V & 90 sh & - & 13 \\
SAX J2103.5+4545 & - & B0V & - & $240 \pm 20$ & 14 \\
\hline
\end{tabular}

Note: [1] Negueruela et al. (2001), [2] Reig et al. (1997), [3] Negueruela et al. (1999) [4] Lyubimkov et al. (1997), [5] Delgado-Martí et al. (2001), [6] Reig et al. (2005) [7] Haigh et al. (2004), [8] Grundstrom et al. (1997), [9] Reig et al. (2001) [10] Janot-Pacheco et al. (1981), [11] Janot-Pacheco et al. (1982), [12] Webster (1974) [13] Parkes et al (1980), [14] Reig et al.] (2004)

Table 5 Disc-loss episodes in Be/X-ray binaries.

\begin{tabular}{lccccccc}
\hline $\begin{array}{l}\text { Source } \\
\text { name }\end{array}$ & $\begin{array}{c}\text { Spectral } \\
\text { type }\end{array}$ & $V_{0}$ & $(B-V)_{0}$ & $\begin{array}{c}\mathrm{EW}(\mathrm{H} \alpha) \\
(\AA)\end{array}$ & $\begin{array}{c}T_{\text {disc }} \\
(\text { year })\end{array}$ & $\begin{array}{c}T_{\text {loss }} \\
(\text { day })\end{array}$ & Reference \\
\hline 4U 0115+63 & B0.2Ve & 15.5 & 1.43 & +2.4 & $3-5$ & 800 & 1,2 \\
X Per & B0Ve & 6.80 & 0.14 & +2.7 & 7 & 800 & 3,4 \\
1A 0535+262 & O9.7IIIe & 9.44 & 0.41 & +2.5 & $4-5$ & 600 & 5,6 \\
RX J0812.4-3114 & B0.2IVe & 12.5 & 0.4 & +1.4 & 4 & - & 7 \\
RX J0440.9+4431 & B0.2Ve & - & - & -0.5 & $>10$ & $<200$ & 8 \\
IGR J06074+2205 & B0.5Ve & - & - & +2.2 & $>5$ & - & 9 \\
SAX J2103.5+4545 & B0Ve & 14.35 & 1.07 & +2.8 & $1.5-2$ & 1000 & 10 \\
\hline
\end{tabular}

Note: [1] Negueruela et al. (2001), [2] Reig et al. (2007), [3] Roche et al. (1997), [4] Clark et al. (2001) [5] Haigh et al. (1999), [6] Clark et al. (1998), [7] Reig et al. (2001), [8] Reig et al. (2005) [9] Reig et al. (2010b), [10] Reig et al. (2010a) 
these two reddening contributions. Although some iterative procedure has been proposed to correct for both circumstellar and interstellar reddening from narrowband photometry (Fabregat \& Torreión 1998) or using infrared colours (Howells et al. 2001; Dougherty et al. 1994), the most reliable measurement of the true photometric colours of a Be star is when the disc contribution from the disc is negligible. That is the reason that disc-loss episodes are so important. The main accepted disc-loss indicator is the $\mathrm{H} \alpha$ line (Fig. 4). When the star loses the disc, the $\mathrm{H} \alpha$ line shows an absorption profile and the X-ray activity ceases or is largely reduced.

Table 5 list the BeXB that have gone through discloss phases. It also shows the typical time scales associated with disc variability and the intrinsic colours of the underlying B star (not corrected for interstellar reddening). $T_{\text {disc }}$ is the typical duration of formation/dissipation of the circumstellar disc and $T_{\text {loss }}$ represents the maximum duration of the disc-loss phase.

\section{Observational evidence of the disc-neutron star interaction}

Be stars may also exist as single objects, i.e., not forming part of an X-ray binary system. There is general consensus that the optical/IR long-term (months to years) variability seen in Be stars is caused by the equatorial disc around the massive star. In BeXB, the Xray variability is attributed to changes in the accretion rate. Since the circumstellar disc constitutes the fuel that powers the X-ray machinery, the X-ray variability can also be ascribed to changes in the disc. The Be star in a BeXB is assumed to have the same physical properties (mass, radius, luminosity) as isolated Be stars. Therefore, any difference in the variability patterns of isolated and BeXB must be the result of different structure and physical properties of the disc (size, density).

The following questions arise: Are the equatorial discs of isolated Be stars different from those of Be stars forming part of an X-ray binary? If yes, what causes this difference?

The obvious difference between an isolated Be star and a BeXB is the presence of a neutron star in the latter. Since the underlying Be star has the same physical characteristics in both type of systems, we conclude that any difference in the properties of the disc in binaries must be attributed to the neutron star.

Traditionally, the interaction between the two components in a BeXB was thought to go into one direction, namely, from the massive companion to the neutron star, through the transfer of mass. Matter from the Be star captured by the neutron star is accreted and powers the X-ray emission. Given the large mass ratio of the two components it was believed that the neutron star exerted little effect on the massive companion. This picture is now changing, as new observational evidence for the interaction on the neutron star and the circumstellar disc emerges.

In this section, I summarise the observational evidence gathered in recent years of how the compact object affects the optical/IR emission properties of a BeXB. The line of reasoning is the following: BeXB and single Be show different optical/IR patterns of variability because the equatorial disc has different physical properties. The disc exhibits different properties because of the presence or absence of an external agent, that is, the neutron star. The neutron star controls somehow the evolution of the disc because it prevents its free expansion and causes truncation.

In searching for observational evidence for the effects that the neutron star produces on the circumstellar disc of the Be star in BeXB, I have followed two different approaches

1. Correlations with the orbital period. Since BeXB are in general moderately eccentric systems, any property that correlates with the orbital period of the system can be interpreted as due to the action of the neutron star. The logic behind this idea is that the shorter the orbital period, the smaller the orbit. Hence circumstellar discs in narrow-obit systems must be more affected by the gravitational pull exerted by the neutron star during each periastron passage than systems with longer orbital periods.

2. Comparison of the variability patterns in isolated Be stars and $B e X B$. If there is no physical difference between the B star in an isolated Be and the B star in a BeXB, and the optical/IR variability is driven by changes in the disc, then any difference in the observational properties of these two types of systems must come from the effect of the neutron star on the disc. The objective here is to search for such differences in the evolutionary patterns of various physical parameters (disc density and radius, the strength and shape of spectral lines, particularly $\mathrm{H} \alpha$, and/or characteristic time scales).

This section begins with a summary of the viscous decretion disc model (see also Sect. 4.1) and it is followed by a compilation of the observational evidence of the interaction of the neutron star with the circumstellar envelope of the Be star.

\subsection{Disc truncation}

The idea of disc truncation in BeXB was first suggested by Reig et al. (1997) from an observational 


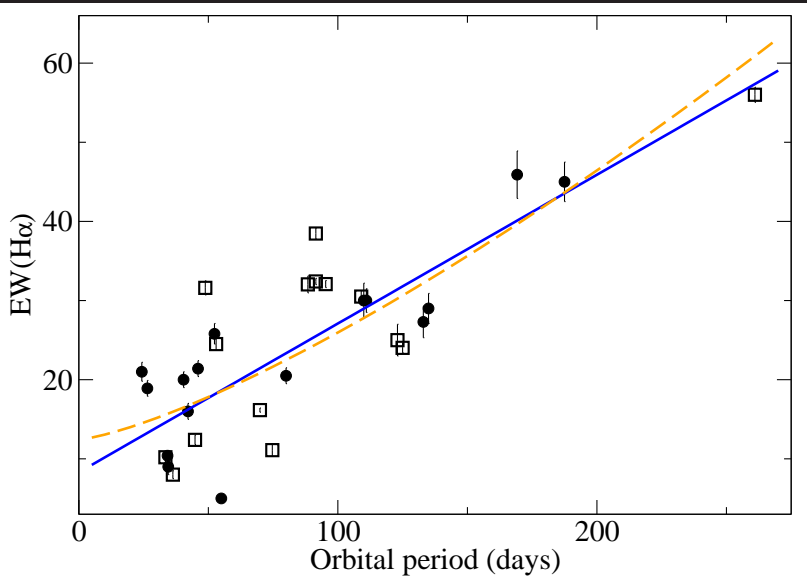

Fig. $15 \mathrm{P}_{\mathrm{orb}}-\mathrm{EW}(\mathrm{H} \alpha)$ diagram. Milky way objects have been represented by circles and BeXB in the Small Magellanic Clouds by squares. The linear regression for the combined data sample is shown with a solid line, while the dashed line represents a power-law fit with exponent $n=4 / 3$.

point of view and the theory subsequently developed by Okazaki and collaborators in a series of papers: Negueruela \& Okazaki (2001); Okazaki \& Negueruela (2001); Okazaki et al. (2002).

The central idea behind the viscous decretion disc models is that Be stars' discs are supported by viscosity. In this respect, the viscous excretion discs are very similar to the well-known viscous accretion discs, except for the changed sign of the rate of the mass flow. Angular momentum is transferred from the optical companion to the inner edge of the disc, increasing its angular velocity to Keplerian (rotationally supported). The radial velocity component is subsonic even at the distance where the neutron star lies. Truncation occurs by the tidal interaction when the resonant torque exerted by the neutron star exceeds the viscous torque. This occurs only at certain radii - where the ratio between the angular frequency of disc rotation and the angular frequency of the mean binary motion is a rational number. The efficiency of truncation depends strongly on the gap, $\delta r$, between the truncation radius and the inner Lagrangian point and the viscosity parameter $\alpha$. If $\tau_{\text {drift }}$ is the time scale for a particle in the disc to cross this gap then truncation will be efficient when $\tau_{\text {drift }}>P_{\text {orb }}$. Typical values of the viscosity parameter are $\alpha<1$. Truncation is expected to be more efficient in low and moderate eccentricity systems $(e \lesssim 0.3)$ with narrow orbits $\left(P_{\text {orb }} \lesssim 40 \mathrm{~d}\right)$ than in high eccentricity systems because the former have wider gaps.

Okazaki et al. (2002) performed numerical simulations of the disc formation around isolated Be stars and of the interaction between the Be-star disc and the neutron star in BeXB and showed that resonant truncation is effective if the viscosity parameter is $\alpha<<1$. There is a radius outside of which the azimuthally-averaged surface density decreases steeply. When $\alpha \sim 0.1$ the slope outside this radius is steeper, giving a stronger truncation effect on the disc. In the truncated disc model, the material lost from the Be star accumulates and the disc becomes denser more rapidly than if around an isolated Be star.

\subsection{Correlations with orbital period}

If the circumstellar disc is responsible for the variability observed at all wavelengths in BeXB, then correlations of an observational parameter with the orbital period can be attributed to the interaction of the neutron star with the disc.

\subsubsection{The $P_{\mathrm{orb}}-E W(H \alpha)$ diagram}

Figure 15 shows an updated version of the $\mathrm{P}_{\text {orb }}{ }^{-}$ $\mathrm{EW}(\mathrm{H} \alpha)$ diagram by Reig et al. (1997, see also Reig (2007); Antoniou et al. (2009b)). The original $\mathrm{P}_{\text {orb }^{-}}$ $\mathrm{EW}(\mathrm{H} \alpha)$ diagram contained $11 \mathrm{BeXB}$, of which only 9 had well established orbital periods. Since then several new BeXB have been discovered and their orbital periods determined. Especially remarkable has been the discovery of a large number of BeXB in the Magellanic Clouds Coe et al. 2005; Liu et al. 2005; Schmidtke \& Cowly 2006; Antoniou et al. 2009b). In this figure, only sources with well-determined orbital periods have been included, thus other SMC X-ray pulsars (such as SXP756, SXP8.80, SXP46.6, and SXP304) with published $\mathrm{H} \alpha$ equivalent widths are not present in the figure.

The $\mathrm{P}_{\text {orb }}-\mathrm{EW}(\mathrm{H} \alpha)$ diagram is based on the fact that the $\mathrm{H} \alpha$ line is the prime indicator of the circumstellar disc state. Although an instantaneous measurement of the $\mathrm{EW}(\mathrm{H} \alpha)$ may not be an effective measurement of the size of the disc, the maximum equivalent width, when monitored during a long length of time (longer than the typical time scales for changes in the circumstellar disc, namely, from few months to a few years) becomes a significant indicator of the size of the disc. Based on interferometric observations, Quirrenbach et al. (1997) and Tycner et al. (2005) have shown that there is a clear linear correlation between the net $\mathrm{H} \alpha$ emission and the physical extent of the emitting region. Also, the observed correlations between the spectral parameters of the $\mathrm{H} \alpha$ line (FWHM, EW, peak separation in double-peak profiles) and the rotational velocity that have been observed in many Be stars are interpreted as evidence for rotationally dominated circumstellar disc (Dachs et al. 1986). In particular, interpreting the peak separation $\left(\Delta_{\text {peak }}\right)$ of the $\mathrm{H} \alpha$ split 
profiles as the outer radius $\left(R_{\text {out }}\right)$ of the emission line forming region (Huang 1972)

$\frac{R_{\mathrm{out}}}{R_{*}}=\left(\frac{2 v \sin i}{\Delta_{\text {peak }}}\right)^{2}$

the radius of the emitting region can be estimated (Hummel \& Vrancken 1995; Jaschek \& Jaschek 2004). $v \sin i$ is the projected rotational velocity of the B star ( $v$ is the equatorial rotational velocity and $i$ the inclination toward the observer). As the $\mathrm{EW}(\mathrm{H} \alpha)$ increases, the peak separation decreases, hence increasing the radius of the $\mathrm{H} \alpha$ emitting region (Dachs 1992; Hanuschik 1988). Single peak profiles typically correspond to larger discs.

Note that the value of the $\mathrm{H} \alpha$ equivalent width of Fig. 15 is the maximum ever reported. Some systems, especially in the SMC systems, have been discovered recently, hence the monitoring of the $\mathrm{H} \alpha$ is necessarily short. For these systems, the value of the $\mathrm{EW}(\mathrm{H} \alpha)$ may not represent a high state of the circumstellar disc. The $\mathrm{EW}(\mathrm{H} \alpha)$ of such systems should be considered as lower limits. This is the case of the galactic BeXB GRO J2058+42 and most likely of the SMC source SXP455 (RX J0101.3-7211).

Assuming then, that $\mathrm{EW}(\mathrm{H} \alpha)$ provides a good measure of the size of the circumstellar disc, Fig. 15] indicates that systems with long orbital periods have larger discs, while narrow orbit systems contain smaller discs. The existence of the above relationship provides a strong argument that the discs are truncated, due to the action of the compact object, to a radius that is a fraction of the semimajor axis. Given the similarity in masses of the optical companions in BeXB, we would expect that the truncation radius $r_{\text {trunc }}$ would scale with the semimajor axis according to Kepler's Third Law as $r_{\text {trunc }} \propto P^{2 / 3}$. Since $\mathrm{EW}(\mathrm{H} \alpha)$ scales as the square of the disc radius all other things being equal (Grundstrom \& Gies 2006), the simple prediction would be $E W(H \alpha) \propto P^{4 / 3}$. The solid line in Fig. 15] corresponds to a simple linear regression fit, while the dashed line represents a power-law fit with exponent equal to $4 / 3$.

\subsection{2 $\mathrm{Be} \longrightarrow \mathrm{B} \longrightarrow$ Be phase}

Column 4 of Table 5 gives the duration of the disc formation/dissipation phase, $T_{\text {disc }}$, of those BeXB for which reliable data exist. The orbital period is given in column 6 . As can be seen there is a good correlation between $T_{\text {disc }}$ and the orbital period. Systems with short orbital periods tend to show faster disc growth and dissipation cycles, while longer time scales are associated with longer orbital periods. This result agrees with the scenario that the Be disc is truncated by the tidal torques from the neutron star, which would be more significant for narrower orbits.

\subsubsection{Long-term X-ray variability}

Reig (2007) investigated the long-term X-ray variability, expressed as the root-mean-square $(\mathrm{rms})$ of the $\mathrm{ASM} / R X T E$ light curves, of a set of BeXB and found that the systems with the larger rms are those harbouring faster rotating neutron stars and those in low eccentric and relatively narrow orbits. The rms amplitude was computed as rms $=\sigma^{2} / \bar{x}^{2}$, where $\bar{x}$ is the mean count rate and $\sigma^{2}=\sigma_{\text {obs }}^{2}-\sigma_{\text {exp }}^{2}$ is the difference between the observed variance, $\sigma_{\mathrm{obs}}^{2}=\sum_{i}\left(x_{i}-\bar{x}\right)^{2} / N$, and the expected variance, $\sigma_{\exp }^{2}=\sum_{i} \sigma_{i}^{2} / N\left(\sigma_{i}\right.$ are the experimental errors, and $N$ is the total number of points).

Figure 16 shows the rms as a function of the spin period, eccentricity and orbital period of the systems. Note that by using the rms the emphasis is put on the amplitude of the variations rather than on how often the variations take place. For the sake of comparison, persistent BeXB and supergiant X-ray binaries, that is, systems whose optical companion is an evolved (luminosity class I-II) star, have also been included. Different type of systems have been represented by different symbols as follows: transient BeXB by circles, persistent BeXB by stars and supergiants by squares. Systems that have shown Type II outbursts are represented by black filled circles. Triangles denote the class of low-e BeXB (Sect. 1.2.1). It is very likely that low-e BeXB and persistent BeXB form only one class of systems. In fact, X-Per, which is considered the prototype of the persistent BeXB, also belongs to the class of low-e BeXB.

In all cases a clear anticorrelation is apparent: the systems with the faster rotating neutron stars and with low eccentricity and narrow orbits are more variable, i.e., present higher $r m s$. This plot then suggests two interesting results. First, since the BeXB and the SGXB occupy clearly distinct regions in Fig. 16. the X-ray variability in high-mass X-ray binaries does not only depend on the physical conditions in the vicinity of the compact object but also on the mass transfer mechanism, i.e., whether a circumstellar disc is present or not. Second, the systems containing fast spinning neutron stars are more likely to exhibit Type II outbursts.

In general, $i$ ) the more eccentric the orbit the lower the variability and ii) type II activity mainly occurs in low-eccentric systems. The low eccentricity class of BeXB follow the general trend in the rms $-P_{\text {spin }}$ and 


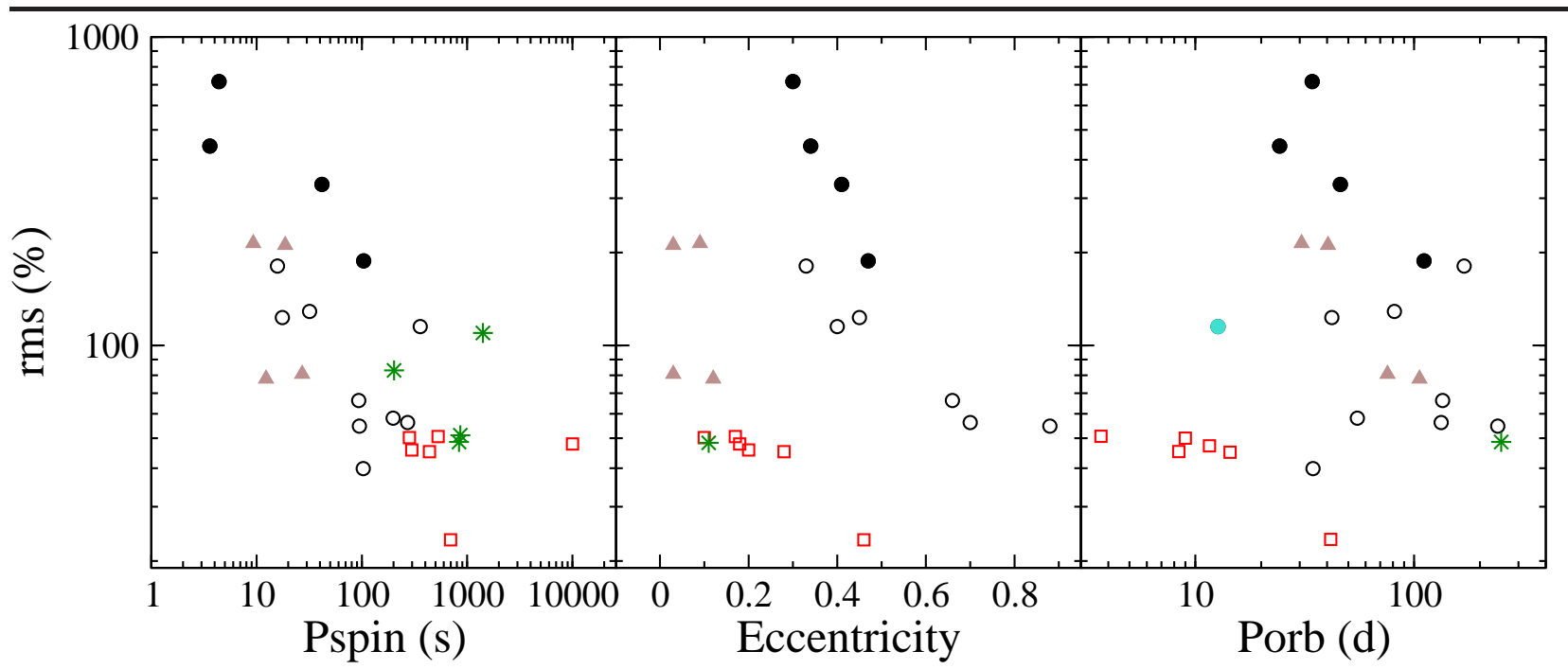

Fig. 16 X-ray rms variability of the $R X T E / A S M$ light curves as a function of the spin period (left), eccentricity (middle) and orbital period (right) of galactic BeXB. Black filled circles denote systems that have shown type II outbursts. Squares represent SGXBs and the star symbols correspond to persistent BeXB. The cyan filled circle on the right panel correspond to SAX J2103.5+4545 (see Sect. 1.1). From Reig (2007).

rms $-P_{\text {orb }}$ plots but clearly distinguish themselves from the classical BeXB, in the rms - e diagram. The anticorrelation of the rms with the orbital period provides further evidence for the truncation of the disc in systems with short orbital periods, i.e., those having narrow orbits.

A prediction of the viscous decretion disc model is that type I outbursts are expected to occur more often in high-eccentric systems, whereas type II outburst would dominate in low-eccentric systems, in agreement with the results shown in Fig. 16.

Coe et al. (2005) carried out a major study of the optical and infrared characteristics of BeXB in the SMC. They found that the optical photometric variability is greatest when the circumstellar disc size is least and also in systems containing fast rotating neutron stars. Reig (2007) extended these results to the Xray domain. While the works by Okazaki \& Negueruela (2001), Zhang et al. (2004) and the $E W(H \alpha)-P_{\text {orb }}$ correlation clearly identify truncation with low eccentric and narrow orbit systems, the plots of the $X$-ray rms as a function of the system parameters (Fig. 16]) and those of the $\mathrm{EW}(\mathrm{H} \alpha)$ and $P_{\text {spin }}$ as a function of the optical photometric rms (Figs 6 and 7 in Coe et al. 2005) identify truncation with variability. That is, systems in which truncation is favoured are the most variable ones in both, the X-ray and optical bands.

\subsection{Be in BeXB versus isolated Be}

The other line of argument to demonstrate that the neutron star exerts a measurable effect on the optical companion is based on the comparison of the variability patterns of the optical/IR emission in isolated Be stars and BeXB.

\subsection{1 $V / R$ variability}

As explained in Sect. 2.1 $\mathrm{V} / \mathrm{R}$ variability is defined as the intensity variations of the two peaks (known as violet and red peak) in the split profile of a spectral line. In many Be stars, if monitored over a long enough period of time, these variations are quasiperiodic Okazaki 1997). $\mathrm{V} / \mathrm{R}$ variability is currently explained by the global one-armed oscillation model. This model suggests that the long-term $\mathrm{V} / \mathrm{R}$ variations are caused by global $m=1$ oscillations in the cool equatorial disc of the Be star. In other words, an enhanced density perturbation develops on one side of the disc, which slowly precesses. The precession time is that associated with the $\mathrm{V} / \mathrm{R}$ quasi-period. The periods of $\mathrm{V} / \mathrm{R}$ variations in isolated Be stars range from years to decades, with a statistical mean of 7 years, which is much longer than the rotational period of the central star (Okazaki 1997). In contrast, in BeXB (Table 6), the $\mathrm{V} / \mathrm{R}$ quasiperiods, $T_{\mathrm{V} / \mathrm{R}}$, are significantly shorter $(\lesssim 5 \mathrm{yr})$, which can be interpreted as the result of smaller discs in BeXB due to truncation caused by the neutron star.

Oktariani \& Okazaki (2009) studied the tidal effect of the companion on the global oscillation modes in equatorial discs around binary Be stars. They found that the oscillation modes are well confined when the disc is larger than a few tens of stellar radii. In smaller discs, however, the mode confinement is incomplete and 


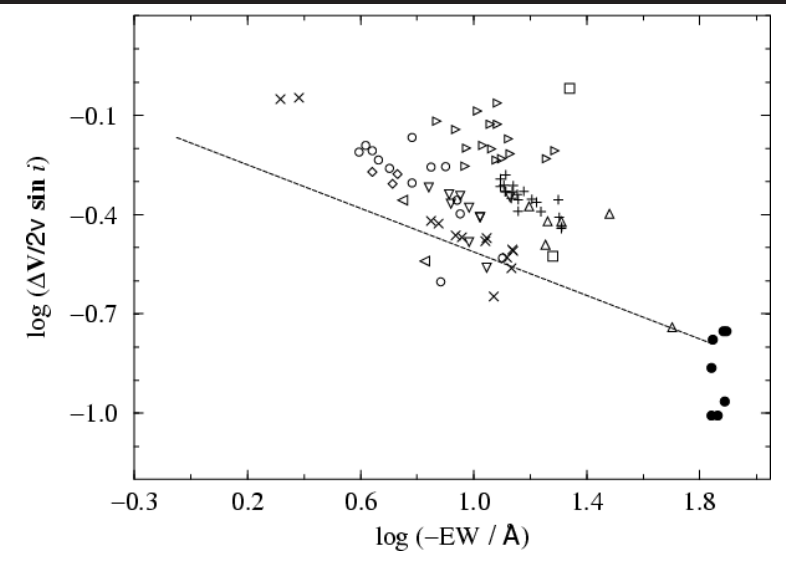

Fig. 17 Peak separation as a function of $\mathrm{EW}(\mathrm{H} \alpha)$. The dashed line represents the average behaviour of the isolated Be stars. Be/X-ray binaries show, on average denser disks. Different sysmbols correspond to different BeXB. From Zamanov et al. (2001).

the oscillation period depends on the binary parameters significantly. The oscillation period becomes longer in the case of a wider binary separation and/or a lower binary mass ratio. Such dependence is observed only for short period binaries. In wide binaries where the disc size is large enough to confine the one-armed modes in the inner part of the disc, the mode characteristics depend little on the binary parameters.

\subsubsection{Disc density}

Zamanov et al. (2001) carried out a comparative study of the circumstellar discs in BeXB and isolated Be stars based on the $\mathrm{H} \alpha$ line emission properties. They found that the discs of BeXB are twice as dense as and have, on average, smaller size than the discs of isolated $\mathrm{Be}$ stars.

Hanuschik (1988, 1989) showed that there is a correlation between the $\mathrm{H} \alpha$ peak separation and the equivalent width in Be stars, according to the law

$\log \left(\frac{\Delta V}{2 v \sin i}\right)=a \log \left(\frac{-E W(\mathrm{H} \alpha)}{\AA}\right)+b$

where $v \sin i$ is the projected rotational velocity and $\mathrm{EW}(\mathrm{H} \alpha)$ is given in Angstroms. $a$ and $b$ are related to the rotational law index $a=-j / 2(j=0.5$ for Keplerian rotation and $j=1$ for conservation of angular momentum) and with the disc electron density, respectively. Figure 17 shows the plot of $\log \frac{\Delta V}{2 v \sin i}$ vs $\log \mathrm{EW}(\mathrm{H} \alpha)$ for a number of BeXB. The straight line $(a=-0.4$, $b=-0.1$ ) represents the average behaviour of the isolated Be stars investigated by Hanuschik (1989). The data points correspond to BeXB. As can be seen, the vast majority of the data points lie above the average line, i.e. they are shifted towards denser discs.

Another result that supports denser disc in Be binaries is the correlation observed between the $\mathrm{H} \alpha$ equivalent width and the $(B-V)$ colour excess in a sample of Be/X-ray binaries in the Magellanic Clouds (Torrejón et al. 2007). The total reddening in the direction of a Be star or BeXB binary is composed of two components: one (constant) produced mainly by dust in the interstellar space through the line of sight $E^{i s}(B-V)$ and another one (variable) produced by the circumstellar gas around the Be star $E^{c s}(B-V)$. The nature and wavelength dependence of these two reddenings is completely different but their final effect upon the photometric indices and spectrum is, in principle, indistinguishable. Torrejón et al. (2007) found that the circumstellar excess is $\sim 10$ times larger for Be stars in binaries than for isolated Be stars with the same amount of emission, and also that the circumstellar colour excess, $E^{c s}(B-V)$ saturates at $\sim 0.2$ magnitudes beyond $\mathrm{EW}(\mathrm{H} \alpha) \approx 20 \AA$.

Again the denser discs seen in BeXB can be explained by disc truncation. Okazaki et al. 2002) showed that during the initial build-up phase, the disc evolution is similar to that for isolated Be stars. But, later on, the effect of the resonant torque becomes apparent, preventing the disc gas from drifting outwards at several resonance radii. As a result, the disc density increases more rapidly than that for isolated Be stars, in agreement with the results presented in this section.

\subsubsection{Disc size}

As mentioned above, interferometric observations have shown that there is a clear linear correlation between the net $\mathrm{H} \alpha$ emission and the physical extent of the emitting region Quirrenbach et al. 1997; Tycner et al. 2005). Using numerical models of the circumstellar discs of Be stars, Grundstrom \& Gies (2006) showed that there are monotonic relationships between the emission-line equivalent width and the ratio of the angular half-width at half maximum of the projected disc major axis to the radius of the star, providing a method to estimate disc radii based only on the $\mathrm{H} \alpha$ equivalent width with an accuracy of about $30 \%$. Since, on average, isolated Be stars show larger $\mathrm{EW}(\mathrm{H} \alpha)$ than $\mathrm{Be}$ stars in BeXB (Reig et al.1997; Antoniou et al. 2009b), we conclude that, on average, the envelopes of Be stars in BeXB are smaller than those present in isolated Be stars.

\subsection{Quantized infrared excess flux states}

Further evidence in favour of the decretion disc model and disc truncation comes from the infrared band. 
Table 6 Characteristic timescales for disc variability in Be/X-ray binaries.

\begin{tabular}{llccccc}
\hline $\begin{array}{l}\text { X-ray } \\
\text { name }\end{array}$ & $\begin{array}{l}\text { Optical } \\
\text { name }\end{array}$ & $\begin{array}{c}\text { Spectral } \\
\text { type }\end{array}$ & $\begin{array}{c}T_{\text {disc }} \\
\text { (year) }\end{array}$ & $\begin{array}{c}T_{\mathrm{V} / \mathrm{R}} \\
\text { (year) }\end{array}$ & $\begin{array}{c}P_{\text {orb }} \\
(\text { day })\end{array}$ & Reference \\
\hline 4U 0115+63 & V635 Cas & B0.2Ve & $3-5$ & $0.5-1.5$ & 24.3 & 1 \\
V 0332+53 & BQ Cam & O9Ve & $4-5$ & 1 & 34.2 & 2,3 \\
4U 0352+309 & X Per & B0Ve & 7 & $0.6-2$ & 250 & 4 \\
1A 0535+262 & V725 Tau & O9.7IIIe & $4-5$ & $1-1.5$ & 111 & 5,6 \\
RX J0812.4-3114 & LS 992 & B0.2IVe & 4 & - & 80 & 7 \\
SAX J2103.5+4545 & - & B0.5V & $1.5-2$ & - & 12.7 & 8 \\
RX J 0146.9+6121 & LS I +61 235 & B1Ve & $>10$ & 3.4 & $>200^{*}$ & 9 \\
4U 1145-619 & V801 Cen & B1Ve & $>10$ & 3 & 186 & 10 \\
RX J0440.9+4431 & LS V +44 17 & B0.2Ve & $>10$ & - & $>150^{*}$ & 11 \\
IGR J06074+2205 & - & B0.5V & $>5$ & $\sim 5$ & - & 12 \\
\hline
\end{tabular}

* Obtained from the $P_{\text {spin }}-P_{\text {orb }}$ correlation.

Note: [1] Negueruela et al. (2001), [2] Negueruela et al. (1999), [3] Goranskii (2001) [4] Clark et al. (2001), [5] Haigh et al. (2004), [6] Clark et al. (1998) [7] Reig et al. (2001), [8] Reig et al. (2010a), [9] Reig et al. (2000) [10] Stevens et al. (1997), [11] Reig et al. (2005), [12] Reig et al. (2010b)

Haigh et al. (2004) showed that the Be/X-ray binary A $0535+26$ displays a clear bimodality in the infrared magnitudes distribution, suggesting that the system alternates between a faint and a bright flux state.

These quantized infrared excess flux states are a natural consequence of the resonant truncation hypothesis proposed by Okazaki \& Negueruela (2001) and constitute a firm observational verification of the proposed resonant truncation scheme for Be X-ray binaries. According to this model, tidal torques, which operate most strongly in orbits resonant with that of the neutron star, truncate the disc at radii meeting the condition $P_{\mathrm{NS}}: P_{\text {trunc }}=n: 1$ where $\mathrm{n}$ is an integer. Haigh et al. (2004) identified the two states with resonances 5:1 and $6: 1$. A possible brighter infrared state corresponding to resonance 4:1 may have also been seen during the 70's.

The simultaneity of X-ray activity with transitions between these states strongly suggests a broad mechanism for outbursts, in which material lost from the disc during the reduction of the truncation radius is accreted by the neutron star.

The idea is simple and neat. When the disc outer radius makes a quantum leap inwards the material present between those radii must be relocated elsewhere in the system and becomes available for accretion. In 1993/94 a change of infrared state as accompanied by a giant X-ray outburst. This simultaneity of the type II outburst with the change of infrared state strongly suggests that the giant outburst was caused by the accretion of material previously resident between the 5:1 and 6:1 resonances. Importantly, truncation would appear to be the cause of the $\mathrm{X}$-radiation and not vice versa, as truncation commenced before X-ray emission.

\section{$6 \mathrm{Be} / \mathrm{X}$ in other galaxies}

The study of X-ray source populations in other galaxies is hampered by the large distance from us. Even other galaxies of the Local Group are too far to reach the quiescence population of HMXBs $\left(L_{X} \sim 10^{33}-10^{35}\right.$ erg $\left.\mathrm{s}^{-1}\right)$. Good enough signal-to-noise optical spectra, needed to derive the spectral type of the optical counterparts, are very hard to achieve. Because of the relatively low intergalactic extinction, well-established distance and their proximity, the Magellanic Clouds (MC) represent the best targets to study a complete X-ray binary population. In fact, they are the only galaxies for which meaningful statistical studies can be performed (current space detectors can detect MC Xray sources down to a few $10^{33} \mathrm{erg} \mathrm{s}^{-1}$ ). Moreover, the $\mathrm{MC}$ show different chemical compositions and are heavily interacting with the Milky Way, which affects their star formation history. Therefore, the study of stellar populations in the $\mathrm{MC}$ is particularly rewarding.

Although past X-ray missions with imaging capabilities, such as Einstein (Wang \& Wu 1992), ROSAT (Haberl et al. 2000), and ASCA (Yokogawa et al. 2003) have surveyed the MC, the deep surveys of the current missions XMM-Newton (Sasaki et al. 2003; Eger \& Haberl 2008), CHANDRA (McGowan et al. 2008), RXTE (Galache et al. 2008) and INTEGRAL (Götz et al. 2006; Coe et al. 2010) complemented with observations from ground-based telescopes in the optical/IR band (Schmidtke \& Cowly 2006; Schurch et al. 2007; McBride et al. 2008; Antoniou et al. 2009a.b) are the ones giving new insights into population synthesis models. 
The Small Magellanic Cloud (SMC) hosts an unexpected large population of HMXBs. Initial estimates of the number of HMXBs in the SMC were based on the mass of the SMC relative to that of the Milky Way. The SMC is a few percent of the mass of the Galaxy, in which about $70 \mathrm{X}$-ray pulsars are known. Therefore one would expect to find only two or three systems. However, there are about 50 HMXBs in the SMC. Curiously, only one (SMC X-1) of these systems is not a $\mathrm{Be} / \mathrm{X}$, that is, it contains a supergiant companion.

The reduced metallicity of the SMC ( $\sim$ one fifth of solar) cannot explain, by itself, the large number of HMXBs in this galaxy (Dray 2006). In addition to reduced metallicity, a large star formation rate is needed to explain this excess of HMXBs (Grimm et al. 2003). The star formation rate/mass of the SMC is 150 times that of the Milky Way. The star formation rate may have been enhanced as a result of tidal interactions between the MC and the Galaxy. However, the most recent close approach of the SMC and LMC was $200 \mathrm{Myr}$ ago - much longer than the evolutionary timescale of $\mathrm{Be} / \mathrm{X}$-ray binaries. This implies that either there has been a significant delay between the encounter of the SMC and LMC and the onset of star formation, or that subsequent waves of star formation have given rise to these Be/X-ray binaries (Harris \& Zaritsky 2004).

The main question that we wish to address in this section is whether the population of BeXB in the MC differs from that of the Milky Way. We focus on the statistical distributions of spin periods, spectral type and $\mathrm{H} \alpha$ equivalent width of the optical counterparts.

\subsection{Spectral-type}

The spectral distribution of the BeXB population in the Milky Way is characterised by a sharp peak at $\mathrm{B} 0$ and a relatively narrow width, namely, no BeXB are found beyond type B2 and earlier than type O8. Negueruela \& Coe (2002); Antoniou et al. (2009b) showed that BeXB in the LMC follow the same distribution as those in our Galaxy, while McBride et al. (2008) and Antoniou et al. (2009b) showed that the spectral distribution of $\mathrm{BeXB}$ in the $\mathrm{SMC}$ is also consistent with that of the Milky Way. The only difference might be the presence of a very small number of BeXB with spectral type later than B2 in the SMC. However, given the very low number of these systems and the uncertainty in the spectral classification, this difference is not statistically significant.

\section{$6.2 \mathrm{H} \alpha$ equivalent width}

On average, the $\mathrm{H} \alpha$ equivalent width $(\mathrm{EW}(\mathrm{H} \alpha))$ of the SMC population of BeXB is larger than that of the

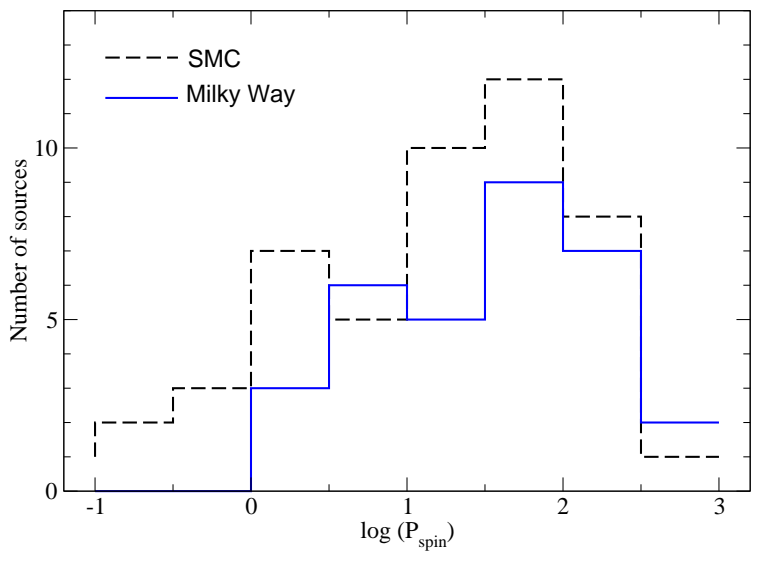

Fig. 18 Distribution of pulse periods in the SMC and the Galaxy.

Milky Way. Antoniou et al. (2009b) found that the $\mathrm{EW}(\mathrm{H} \alpha)$ distribution of SMC BeXB peaks in the interval from -15 to $-25 \AA$, while the $\mathrm{EW}(\mathrm{H} \alpha)$ of Galactic BeXB distribute more uniformly in the interval from -30 to $0 \AA$. Likewise, the number of sources with $\mathrm{EW}(\mathrm{H} \alpha)>50 \AA$ is larger in the SMC. For the LMC systems the sample is too small to draw any conclusions. This trend may just be a selection effect. The discovery of BeXB in the SMC results mainly from X-ray observations. Due to their larger distance we tend to detect the brightest systems, hence those with a large reservoir of accreting material, i.e., those with larger discs.

\subsection{Spin periods}

As indicated in Sect. 2 virtually all HMXBs are X-ray pulsars. Histograms of the spin period of HMXBs in the MC and their comparison with Galactic sources can be found in Laycock et al. (2005) and Liu et al. (2005). They both find that the SMC population appears to be shifted to shorter periods. However, while this shift is very significant in the Lavcock et al. (2005) sample, the difference is minor in the Liu et al. (2005) sample. One contribution to the difference between the Galactic and SMC pulse period distributions is the apparent lack of supergiant wind accretion pulsars in the SMC. HMXBs with supergiant companions tend to have longer $(>100 \mathrm{~s})$ spin periods than BeXB. As stated before only one such system is known in the SMC. Figure 18 shows the spin period distributions of well established BeXB (i.e. excluding supergiant wind-powered systems) in the SMC and Milky Way from the lists compiled by Laycock et al. (2005) and Raguzova \& Popov (2005). A Kolmogorov-Smirnov test gives no real evidence against the null hypothesis that the two samples are drawn from the same distribution. 


\section{Summary}

The traditional picture of two classes of high-mass $\mathrm{X}$-ray binaries, namely supergiant X-ray binaries and $\mathrm{Be} / \mathrm{X}$-ray binaries is giving way to a more complex situation where newly discovered systems may not fit in these categories. Superfast X-ray transients, low eccentricity BeXB, $\gamma$-ray binaries, and $\gamma$-Cas like objects are among the new type of systems that only recently have begun to emerge. BeXB do not form a homogeneous group either with transient and persistent sources, highly eccentric and nearly circular orbits, and fast $\left(\mathrm{P}_{\text {spin }} \sim\right.$ few seconds $)$ and slow $\left(\mathrm{P}_{\text {spin }} \sim\right.$ few hundred of seconds) rotating neutron stars.

Virtually, all BeXB are X-ray pulsars. The study of the variability of the X-ray spectral and pulse timing parameters across giant outbursts is crucial to understand the physics of accretion in strongly magnetised neutron stars. Only recently, attention is being paid to the aperiodic variability and evolution of broadband noise components across the outburst. The application of diagrams such as hardness-intensity and colour-colour diagrams, so widely used in black-hole and low-mass binaries, to BeXB and the definition of spectral states is a promising way to understand the phenomenology of the evolution of outbursts in these systems.

Progress in understanding BeXB is also being made in the optical and infrared bands where the massive companion shines bright. The data gathered over the past ten years indicate that the interaction between the compact object and the Be type star works in two directions: the massive companion provides the source of matter for accretion; the amount of matter captured and the way it is captured (transfer mechanism) change the physical conditions of the neutron star (e.g. by spinning it up or down and shrinking or expanding the magnetosphere). But also, the continuous revolution of the neutron star around the optical counterpart produces observable effects, the most important of which is the truncation of the Be star's equatorial disc.

Future missions with improved sensitivities in the Xray band will help solve remaining open questions such as the formation of accretion discs during X-ray outbursts, the origin of quasi-periodic oscillations, characterisation of the population of persistent BeXB and the triggering mechanism of the giant outbursts.

\section{Acknowledgements}

I want to thank the referee, Douglas Gies, for his careful reading of this manuscript and his useful and important comments. This work has been supported by the European Union Marie Curie grant MTKD-CT2006-039965 and EU FP7 "Capacities" GA No206469. 


\section{References}

Antoniou, V., Zezas, A., Hatzidimitriou, D., \& McDowell, J.C., 2009a, ApJ, 697, 1695

Antoniou, V., Hatzidimitriou, D., Zezas, A., \& Reig, P., 2009b, ApJ, 707, 1080

Atoyan, A. M., \& Aharonian, F. A., 1999, MNRAS, 302, 253

Balona, L.A. 2000, in The Be phenomenon in early-type stars, IAU Colloq. 175, p.1, Eds. Smith, M., Henrichs, H.F., \& Fabregat, J., ASPC, 214, 1

Belczynski, K., \& Ziolkowski, J., 2009, ApJ, 707, 870

Belloni, T., \& Hasinger, G. 1990, 230, 103

Bildsten, L., Chakrabarty, D., Chiu, J., Finger, M. H., Koh, D. T., et al. 1997, ApJS, 113, 367

Bird, A. J., Bazzano, A., Bassani, L., Capitanio, F., Fiocchi, M., Hill, A. B., Malizia, A., McBride, V. A., Scaringi, S., Sguera, V., et al. 2010, ApJS, 186, 1

Bjorkman, J.E., \& Cassinelli, J.P., 1993, ApJ, 409, 429

Blay, P., Negueruela, I., Reig, P., Coe, M. J., Corbet, R. H. D., Fabregat, J., \& Tarasov, A. E., 2006, A\&A, 446, 1095

Bodaghee, A., Walter, R., Zurita-Heras, J. A., et al. 2006 , A\&A, 447, 1027

Bosch-Ramón, V., Aharonian, F:A., \& Paredes, J.M., 2005, A\&A, 432, 609

Brown, J. C., Cassinelli, J. P., \& Maheswaran, M., 2008, ApJ, 688, 132

Burderi, L., Robba, N.R., La Barbera, N., Guainazzi, M. 1997, ApJ, 481, 943

Casares, J., Ribas, I., Paredes, J. M., Martí, J., Allende Prieto, C. 2005a, MNRAS, 360, 1105

Chaty, S., 2008, ChJAS, 8, 197

Chernyakova, M., Neronov, A., \& Walter, R., 2006, MNRAS, 372, 1585

Clark, J. S., Tarasov, A. E., Steele, et al. 1998, MNRAS, 294, 165

Clark, J. S. Steele I.A., 2000, A\&AS, 141, 65

Clark, J. S., Tarasov, A. E., Okazaki, A. T., Roche, P., Lyuty, V. M. 2001, 380, 615

Coe, M. J., Roche, P., Everall, C., Fishman, G. J., Hagedon, K. S., Finger, M., Wilson, R. B., Buckley, D. A. H. et al.

Coe, M. J., Edge, W. R. T., Galache, J. L., \& McBride, V. A., 2005, MNRAS, 356, 502

Coe, M.J., Bird, A.J., Buckley, D,A.H., Corbet, R.H.D., Dean, A.J. et al. 2010, MNRAS, in press, arXiv:1004.2219

Cohen, D. H., Hanson, M. M., Townsend, R. H. D., Bjorkman, K. S., \& Gagé, M., 2005, in The Nature and Evolution of Disks Around Hot Stars, ASP Conference Series, Vol. 337, astro-ph/0410317

Collins, George W., \& Truax, Ryland J., 1995, ApJ, 439, 860

Corbet, R. H. D., 1986, MNRAS, 220, 1047

Cranmer, S. R., 2005, ApJ, 634, 585

Cranmer, S. R., 2009, ApJ, 701, 396

Dachs, J. \& Wamsteker, W. 1982, A\&A, 107, 240

Dachs, J., Hanuschik, R.W., Kaiser, D., \& Rohe, D. 1986, A\&A, 159, 276

Dachs, J., Engels, D., \& Kiehling, R. 1988, A\&A, 194, 167

Dachs, J., Hummel, W., \& Hanuschik, R.W. 1992, A\&ASS, 95, 437

Dai, H.-L. Liu, X.-W., \& Li, X.-D. 2006, ApJ, 653, 1410
Davidson, K., \& Ostriker, J.P., 1973, ApJ, 179, 585

Davies, R. E., Fabian, A. C., \& Pringle, J. E. 1979, MNRAS, 186,779

Davies, R. E., \& Pringle, J. E. 1981, MNRAS, 196, 209

Delgado-Martí, H., Levine, A. M., Pfahl, E., Rappaport, S. A., 2001, ApJ, 546, 455

Dhawan, V., Mioduszewski, A., \& Rupen, M., 2006, in Proceedings of the VI Microquasar Workshop: Microquasars and Beyond, September 18-22, Como, Italy, 52

Dougherty, S. M.; Waters, L. B. F. M., Burki, G., Cote, J., Cramer, N., van Kerkwijk, M. H., \& Taylor, A. R., 1994, A\&A, 290, 609

Dray, L.M., 2006, MNRAS, 370, 2079

Dubus, G., 2006, A\&A, 456, 801

Eger, P. \& Haberl, F., 2008, A\&A, 491, 841

Fabregat, J. \& Torrejón, J.M., 1998, A\&A, 332, 643

Finley, J. P., Taylor, M., \& Belloni, T. 1994, ApJ, 429, 356

Galache, J. L., Corbet, R. H. D., Coe, M. J., Laycock, S., Schurch, M. P. E., Markwardt, C., Marshall, F. E., Lochner, J., 2008, ApJS, 177, 189

Gehrz, R. D., Hackwell, J. A., \& Jones, T. W. 1974, ApJ, 191,675

Georganopoulos, M., Aharonian, F. A., \& Kirk, J. G. 2002, A\&A, 388, L25

Ghosh,P. \& Lamb, F.K., 1979, ApJ, 234, 296

Gies, D.R., Bagnuolo, W.G., Jr., Ferrara, E.C., Kaye, A.B., Thaller, M.L., Penny, L.R., \& Peters, G.J., 1998, ApJ, 493, 440

Gies, D.R., 2000, in IAU Coll. 175: The Be Phenomenon in Early- Type Stars, ASP Conf. Ser., 214, 668

Gies, D. R., Bagnuolo, W. G., Jr., Baines, E. K., ten Brummelaar, T. A., Farrington, C. D., et al., 2007, ApJ, 654, 527

Goranskii, V. P. 2001, AstL, 27, 516

G̈̈z, D., Mereghetti, S., Merlini, D., Sidoli, L., \& Belloni, T. 2006, A\&A, 448, 873

Grebel, E. K., Richtler, T., \& de Boer, K. S., 1992, A\&A, $254, \mathrm{~L} 5$

Grebenev, S.A., 2010, in Proceedings of the Workshop The Extreme sky: Sampling the Universe above $10 \mathrm{keV}$, Otranto, Italy, PoS, 96, 60

Grimm, H.-J., Gilfanov, M., \& Sunyaev, R., 2003, MNRAS, 339,793

Grundstrom, E.D., Boyajian, T.S., Finch, C. et al. 2007, ApJ, 660, 1398

Grundstrom, E.D., \& Gies, D.R., 2006, ApJ, 651, L53.

Grundstrom, E. D., Boyajian, T. S., Finch, C., Gies, D. R., Huang, W., et al. 2007, ApJ, 660, 1398

Gutiérrez Soto, J., 2006, PhD "Non-radial pulsations in Be stars: preparation of the COROT space mission", University of Valencia

Haberl, F., Filipović, M. D., Pietsch, W., \& Kahabka, P., 2000, A\&AS, 142, 41

Haigh, N. J., Coe, M. J., Steele, I. A., \& Fabregat, J. 1999, MNRAS, 310, L21

Haigh, N. J., Coe, M. J., Fabregat, J. 2004, MNRAS, 350, 1457

Hanuschik, R.W. 1986, A\&A, 166, 185

Hanuschik, R.W., Kozok, J.R., \& Kaiser, D. 1988, A\&A, 189, 147

Hanuschik, R.W. 1989, Ap\&SS, 161, 61 
Hanuschik, R.W., Hummel, W., Dietle, O., \& Sutorius, E. 1995, A\&A, 300, 163

Hanuschik, R.W., 1996, 308,170

Hayasaki, K. \& Ozakaki, A.T. 2004, MNRAS, 350, 971

Huang, S.S. 1972, ApJ, 171, 549

Harris, J., \& Zaritsky, D., 2004, AJ, 127, 1531

Howells, L., Steele, I.A., Porter, J.M., \& Etherton, J., 2001, A\&A, 369, 99

Hummel, W. 1994, A\&A, 289, 458

Hummel, W., \& Vrancken, M. 1995, 302, 751

Hummel, W., \& Hanuschik, R.W. 1997, A\&A, 320, 852

Ikhsanov, N.R., 2001a, 368, L5

Ikhsanov, N.R., 2001b, 375, 944

Ikhsanov, N.R., 2007, MNRAS, 375, 698

James, M., Paul, B., Devasia, J., \& Indulekha, K., 2010 MNRAS, 407, 285

Janot-Pacheco, E., Ilovaisky, S. A., \& Chevalier, C. 1981, A\&A, 99, 274

Janot-Pacheco, E., Chevalier, C., \& Ilovaisky, S.A., 1982, IAUS, 98, 151

Jaschek, C., \& Jaschek, M. 2004yCat, 6057, 0. VizieR Online Data Catalog: VI/57. Originally published in: 1993 , A\&AS, 97, 807

Kaufman Bernadó, M. M., Romero, G. E., \& Mirabel, I. F. 2002, A\&A, 385, L10

Kaur, R., Paul, B., Kumar, B., \& Sagar, R., 2008, MNRAS, 386,2253

Koenigsberger, G., Georgiev, L., Moreno, E., Richer, M.G., Toledano, O., Canalizo, G., \& Arrieta, A., 2006, A\&A 458,513

Kriss, G. A., Cioffi, D. F., Canizares, C. R. 1983, ApJ, 272, 439

Kylafis, N.D., Papadakis, I.E., Reig, P., Giannios, D., \& Pooley, G.G., 2008, A\&A, 489, 481

La Palombara, N., Sidoli, L., Esposito, P., Tiengo, A., \& Mereghetti, S., 2009, A\&A, 505, 947

Laycock, S., Corbet, R. H. D., Coe, M. J., Marshall, F. E., Markwardt, C., Lochner, J., 2005, ApJS, 161, 96

Lazzati, D. \& Stella, L., 1997, ApJ, 476, 267

Lee, U., Saio, H., \& Osaki, Y., 1991, MNRAS, 250, 432

Liu, Q. Z., van Paradijs, J., van den Heuvel, E. P. J. 2005, A\&A, 442, 1135

Liu, Q. Z., van Paradijs, J., van den Heuvel, E. P. J., 2006, A\&A, 455, 1165

Liu, Q. Z., van Paradijs, J., van den Heuvel, E. P. J., 2007, A\&A, 469, 807

Lopes de Oliveira, R., Motch, C., Haberl, F., Negueruela, I., \& Janot-Pacheco, E., 2006, A\&A, 454, 265

Lucy, L.B. \& Solomon, P.M. 1970, ApJ, 159, 879

Lyubimkov, L. S., Rostopchin, S. I., Roche, P., \& Tarasov, A. E., 1997, MNRAS, 286, 549

Maintz, M., Rivinius, T., Stahl, O., Stefl, S., \& Appenzeller, I. 2005, Publ. Astron. Inst. Czechoslovakia, 93, 21

Markoff, S., Nowak, M., Corbel, S., Fender, R., \& Falcke, H., 2003, A\&A, 397, 645

McBride, V. A., Coe, M. J., Negueruela, I., Schurch, M. P. E., McGowan, K. E., 2008, MNRAS, 388, 1198

McGowan, K. E., Coe, M. J., Schurch, M. P. E., McBride, V. A., Galache, J. L., Edge, W. R. T., Corbet, R. H. D., Laycock, S., Buckley, D. A. H., 2008, MNRAS, 383, 330

McSwain, M. V., \& Gies, D. R., 2005, ApJ, 622, 1052
Moffat, A. F. J., Haupt, W., \& Schmidt-Kaler, T., 1973, A\&A, 23, 433

Motch, C., Stella, L., Janot-Pacheco, E., \& Mouchet, M. 1991, ApJ, 369, 490

Negueruela, I., Roche, P., Fabregat, J., Coe, M. J. 1999, MNRAS, 307, 695

Negueruela, I., \& Okazaki, A. T., 2001, A\&A, 369, 108

Negueruela, I., Okazaki, A. T., Fabregat, J., Coe, M. J., Munari, U., Tomov, T. 2001, A\&A, 369, 117

Negueruela, I., \& Coe, M. J., 2002, A\&A, 385, 517

Negueruela, I., Smith, D. M., Reig, P., Chaty, S., \& Torrejn, J. M., 2006, ESASP, 604, 165

Negueruela, I. \& Schurch, M. P. E., 2007, A\&A, 461, 631

Negueruela, I., Torrejón, J.M., Reig, P., Ribó, M., Smith, D. M., 2008, AIPC, 1010, 252

Nespoli, E.,2010, in Multiwavelength study of accretionpowered pulsars, $\mathrm{PhD}$ dissertation thesis. University of Valencia (Spain).

Neto, T.V., \& de Freitas Pacheco, S.J.C., 1982, MNRAS, 198,659

Okazaki A.T. 1991, PASJ 43, 7

Okazaki A.T. 1996, PASJ, 48, 305

Okazaki A.T. 1997, A\&A, 318, 548

Okazaki A.T. 2001, PASJ, 53, 119

Okazaki, A.T., \& Negueruela, I. 2001, A\&A, 377, 161

Okazaki, A. T., Bate, M. R., Ogilvie, G. I., \& Pringle, J. E., 2002, MNRAS, 337, 967

Oktariani, F., \& Okazaki, A.T., 2009, PASJ, 61, 57

Owocki, S. P., Cranmer, S. R., \& Gayley, K. G, 1996, ApJ, 472, L115

Papaloizou, J.C., Savonije, G. J., Henrichs, H. F. 1992, A\&A, 265, L45

Paredes, J.M., 2008, AIPC, 1085, 157

Parkes, G. E., Murdin, P. G., \& Mason, K. O., 1980, MNRAS, 190, 537

Peters, G. J., Gies, D. R., Grundstrom, E. D., \& McSwain, M. V., 2008, ApJ, 686, 1280

Pfahl, E., Rappaport, S., Podsiadlowski, P., Spruit, H. 2002, ApJ, 574, 364

Podsiadlowski, Ph., Rappaport, S., \& Han, Z., 2003, MNRAS, 341, 385

Porter, J.M., 1996, MNRAS, 280, L31

Porter, J.M., 1997, A\&A, 324, 597

Porter, J. M. \& Rivinius, T. 2003, PASP, 115, 1153

Quirrenbach, A., Bjorkman, K. S., Bjorkman, J. E. 1997, ApJ, 479, 477

Raguzova, N. V., \& Popov, S. B., 2005, A\&AT, 24, 151

Reig, P., Fabregat, J., Coe, M. J. 1997, A\&A, 322, 193

Reig, P. \& Roche, P. 1999, MNRAS, 306, 100

Reig, P., Negueruela, I., Coe, M. J. et al. 2000, MNRAS, 317, 205

Reig, P., Negueruela, I., Buckley, D. A. H., Coe, M. J., Fabregat, J., Haigh, N. J. 2001, A\&A, 367, 266

Reig, P., Negueruela, I., Fabregat, J., Chato, R., Blay, P., \& Mavromatakis, F., 2004, A\&A, 421, 673

Reig, P., Negueruela, I., Fabregat, J., Chato, R., Coe, M. J. 2005, A\&A, 440, 1079

Reig, P., Larionov, V., Negueruela, I., Arkharov, A. A., Kudryavtseva, N. A. 2007, A\&A, 462, 1081

Reig, P., 2007, MNRAS, 377, 867

Reig, P., 2008, A\&A, 489, 725 
Reig, P., Torrejón, J.M., Negueruela, I., Blay, P., Ribó, M. Wilms, J. 2008, A\&A, 2009, A\&A, 494, 1073

Reig, P., S owikowska, A., Zezas, A., \& Blay, P., 2010, MNRAS, 401, 55

Reig, P., Zezas, A., \& Gkoubelis, L., 2010, A\&A, in press

Rivinus, Th., Stefl, S., Maintz, M., Stahl, O. \& Baade, D., 2004, A\&A, 427, 307

Rivinus, Th., Stefl, S., \& Baade, D., 2006, A\&A, 459, 137

Robinson, R. D., Smith, M. A., \& Henry, G.W., 2002, ApJ, 575, 435

Roche, P., Larionov, V., Tarasov, A. E. et al. 1997, A\&A, 322, 139

Romano, P., Sidoli, L., et al. 2010, arXiv1001.2439

Romero, G.E., Torres, D.F., Kaufman Bernad, M.M., \& Mirabel, I.F., 2003, A\&A, 410, L1

Sasaki, M., Pietsch, W., \& Haberl, F., 2003, A\&A, 403, 901

Savonije G.J. \& Heemskerk M.H.M. 1993, A\&A, 276, 409

Schmidtke, P.C. \& Cowly, A.P. 2006, ApJ, 132, 919

Schurch, M.P.E., Coe, M.J., McGowan, K.E., McBride, V.A., Buckley, D. et al. 2007, MNRAS, 381, 1561

Silaj, J., Jones, C. E., Tycner, C., Sigut, T. A. A., \& Smith, A. D., 2010, ApJS, 187, 228

Slettebak, A. 1982, ApJS, 50, 55

Slettebak, A. 1988, PASP, 100, 770

Smith, D. M., Heindl, W.A., Markwardt, C.A., et al. 2006, ApJ, 638, 974

Steele,I.A., Negueruela, I., \& Clark, J.S., 1999, A\&AS, 137, 147

Stella, L., White, N. E., \& Rosner, R., 1986, ApJ, 308, 669

Stevens, J. B., Reig, P., Coe, M. J., et al. 1997, MNRAS, 288,988

Telting, J. H., Heemskerk, M. H. M., Henrichs, H. F., \& Savonije, G. J. 1994, A\&A, 288, 558

Torrejón, J. M., Negueruela, I., \& Riquelme, M. S., 2007, ASPC, 361, 503

Torres, D., 2010, Proceedings of the 1st Sant Cugat Forum on Astrophysics, "ICREA Workshop on the high-energy emission from pulsars and their systems", arXiv:1008.0483

Townsend, R. H. D., Owocki, S. P., \& Howarth, I. D., 2004, MNRAS, 350, 189

Tycner, C., Lester, J.B., Hajian, A.R., et al. 2005, ApJ, 624, 359

van der Klis, M. 2006, in X-ray sources Binaries, eds. W.H.G. Lewin \& J. van Paradijs, E.P.J. van den Heuvel, 1995 Cambridge University Press, p.252

van der Klis, M. 2006, in Compact Stellar X-ray sources, eds. W.H.G. Lewin \& M. van der Klis, Cambridge University Press, p.39

Walter, R. \& Zurita Heras, J. 2007, A\&A, 476, 335

Wang, Q, \& Wu, X., 1992, ApJS, 78, 391

Wang, W, 2009, A\&A, arXiv0912.0337

Waters, L.B.F.M., \& van Kerkwijk, M.H., 1989, A\&A, 223, 196

Webster, B. L., 1974, MNRAS, 169, 53

Williams, S.J., Gies, D.R., Matson, R.A., Touhami, Y., Grundstrom, E.D., Huang, W., \& McSwain, M.W., 2010, arXiv:1009.4947

Wilson, C. A., Finger, M. H., \& Camero-Arranz, A., 2008, ApJ, 678, 1263
Woolf, N. J., Stein, W. A., \& Strittmatter, P. A. 1970, A\&A, 9, 252

Yokogawa, J., Imanishi, K., Tsujimoto, M., Koyama, K., \& Nishiuchi, M., 2003, PASJ, 55, 161

Yudin, R.V., 2001, A\&A, 368, 912

Zamanov, R. K., Reig, P., Mart, J., Coe, M. J., Fabregat, J., Tomov, N. A., \& Valchev, T. 2001, A\&A, 367, 884

Zhang, F., Li, X.D., \& Wang, Z.R. 2004a, ApJ, 603, 663

Zhang, F., Li, X.D., \& Wang, Z.R., 2004b, Chin. J. Astron. Astrophy., 4, 320

Zurita Heras, J. A., de Cesare, G., Walter, R., et al. 2006, A\&A, 448, 261 